\title{
A framework for optimal actuator/sensor selection in a control system
}

\author{
Ahmadreza Argha ${ }^{1}$, Steven W. Su ${ }^{2 *}$ \\ Andrey Savkin ${ }^{1}$, and Branko Celler ${ }^{1}$ \\ ${ }^{1}$ School of Electrical Engineering and Telecommunications, \\ University of New South Wales, \\ Sydney, NSW 2052 Australia. \\ ${ }^{2}$ Faculty of Engineering and Information Technology, \\ University of Technology Sydney, \\ Sydney, NSW 2007, Australia.
}

August 29, 2017

\begin{abstract}
When dealing with large-scale systems, manual selection of a subset of components (sensors/actuators), or equivalently identification of a favourable structure for the controller, that guarantees a certain closedloop performance, is not very feasible. This paper is dedicated to the problem of concurrent optimal selection of actuators/sensors which can equivalently be considered as the structure identification for the controller. In the context of a multi-channel $\mathscr{H}_{2}$ dynamic output feedback controller synthesis, we formulate and analyse a framework in which we incorporate two extra terms for penalising the number of actuators and sensors into the variational formulations of controller synthesis problems in order to induce a favourable controller structure. We then develop an explicit scheme as well as an iterative process for the purpose of dealing with the multiobjective problem of controller structure and control law co-design. It is also stressed that the immediate application of the proposed approach lies within the fault accommodation stage of a fault tolerant control scheme. By two numerical examples, we demonstrate the remarkable performance of the proposed approach.
\end{abstract}

keywords Simultaneous actuator/sensor selection, regularisation, row/columnwise sparsification, linear matrix inequality, dynamic output feedback.

${ }^{*}$ Corresponding author. Email: Steven.Su@eng.uts.edu.au 


\section{Introduction}

Actuator/sensor selection problem is a standard step of control system design which aims at selecting an appropriate number, place, and type of actuators/sensors (Van De Wal \& De Jager, 2001). While actuator/sensor selection is, broadly speaking, carried out prior to the physical realisation of the plant, sometimes actuator/sensor selection can be performed for a system with pre-equipped actuators/sensors (Van De Wal \& De Jager, 2001). Roughly speaking, controllers exploiting all available actuation and sensing resources can achieve better closedloop performance compared to the ones that use only a small number of actuation and sensing resources. However, due to the high level of the control system complexity as well as the costs of operation and maintenance, employing controllers with a dense structure does not seem very practical (Dhingra, Jovanović, \& Luo, 2014). Further, in some cases, all existing components cannot be exploited at the same time, e.g. when a plant needs different set of instrumentations at startup and at full load modes (Van De Wal \& De Jager, 2001). Furthermore, the number of components (actuators or sensors) in modern control systems can be very large. Therefore, it is not very feasible to manually find a subset of all available components to meet a specific control objective. Hence, the problem of selecting a configuration of actuators (sensors) from the set of all available actuators (sensors), while the control performance remains at an acceptable level compared to the non-sparse performance, is a well-known problem in the literature of control theory; see for example Joshi and Boyd (2009); Polyak, Khlebnikov, and Shcherbakov (2013); Rogers (2000); Roy, Chepuri, and Leus (2013); Savkin and Evans (2002); Savkin, Evans, and Skafidas (2001).

There are a large number of investigations considering this problem, but most of them do not consider dynamical systems and/or do not use a systematic method. Joshi and Boyd (2009) considers the problem of sensor selection through a convex optimisation scheme and for a system with linear measurements. Moreover, a method is proposed in Roy et al. (2013) for identifying favourable subsets of available system sensors while minimising the CramerRao bound of a class of nonlinear measurement models. Kekatos, Giannakis, and Wollenberg (2012) formulates the problem of Phasor Measurement Units (PMUs) placement in power systems as a variation of the optimal experiment design. A genetic algorithm-based method is proposed in Rogers (2000) for the problem of actuator selection. Moreover, the problem of simultaneous sensor and actuator placement is considered in Güney and Eşkinat (2008); Nestorović and Trajkov (2013) employing heuristic optimisation-based methods. In Polyak et al. (2013), an LMI approach is utilised for addressing this problem by incorporating certain forms of sparsity in the feedback gain. Further, an ADMM algorithm based method is proposed in Dhingra et al. (2014) which addresses the problems of sensor and actuator selection separately. The problem of actua-

tor/sensor selection for aeroservoelastic systems is considered in Moreno, Pfifer, and Balas (2015), by proposing a heuristic approach that allows a fair comparison between different configurations of sensors/actuators. In Westermayer, Schirrer, Hemedi, and Kozek (2009), a sensor/actuator placement criterion is 
proposed that is able to evaluate the system response intensity using the $\mathscr{H}_{2}$ norm properties of flexible structures, while the balance between low and high frequency modes is guaranteed by the geometric mean of the gramian eigenvalues. It is worth noting that a large number of early methods proposed for the actuator placement problem have been constructed based on open-loop considerations to guarantee that the necessary controllability, reachability or power factor requirements are satisfied (Van De Wal \& De Jager, 2001). The problem of finding the optimal location of sensors/actuators to achieve reduction of the noise field in an acoustic cavity is also considered in Fahroo and Demetriou $(2000)$, in which two control strategies are proposed for this goal. Each strategy indeed optimises an appropriate quadratic performance criterion representing the location of the actuators and/or the sensors.

Basically, to address an actuator/sensor selection problem, one has to, in worst case, check all actuator/sensor sets, implying an exhaustive search for a number of configurations that can grow exponentially with the number of actuators and sensors. This is practically intractable and impossible to perform. As explained in Van De Wal and De Jager (2001), to avoid performing an exhaustive search, a trade-off can be made either in the choice of the search strategy or in the choice of the selection criterion. Another alternative to avoid solving a combinatorial problem is to consider a multi-objective problem of controller structure and control law co-design by incorporating secondary cost functions, which promotes sparsity of the controller, into a main cost function, which represents a performance specification of the closed-loop system (see e.g. Lin, Fardad, and Jovanovic (2011) and Schuler, Münz, and Allgöwer (2014)).

The so-called reweighted $\ell_{1}$ (REL1) norm, known as a convex relaxation of the $\ell_{0}$-norm, is usually exploited to make a convex problem rather than the original combinatorial optimisation problem. The weights (entries of the weighting matrix) are then updated at each step inversely proportional to the strength of individual entries of feedback gain in the previous step. This scheme successively applies to the applications that the sparsity is required to be achieved at the entry-wise level, i.e. minimising the number of communication links in distributed control networks exploiting the so-called bilateral communication scheme (Staroswiecki \& Amani, 2014). However, in cases where the sparsity is noted at a group (e.g. row or column) level, the strength of groups of variables (entries of feedback gain) needs to be considered. We consider $\|\cdot\|_{\text {row }-\ell_{0}}$ $\left(\|\cdot\|_{\text {col }-\ell_{0}}\right)$, that counts the number of nonzero rows (columns) of a matrix, as the row (column) sparsity promoting penalty function and then propose its reweighted convex relaxation. This paper then develops an iterative algorithm, using the relaxed row/column sparsity promoting penalty functions, which is able to penalise the number of sensors and actuators employed in the control system simultaneously.

Most of the sparse control design approaches in the literature have focused on the problem of sparse state feedback and static output feedback design. This paper instead utilises a dynamic output feedback (DOF) scheme to control the system using the measured system outputs. To the best knowledge of authors, concurrent actuator and sensor selection problem through DOF has not 
been researched thoroughly. Furthermore, it should be noted that the sparse row/column-wise feedback design using DOF is a totally different problem compared to the case of row (column) sparse static output (state) feedback design, as the sparsification procedure should be applied only to a certain part of the controller. One immediate application of this approach will be in over-actuated (over-sensed) systems (Casavola \& Garone, 2010, Cristofaro \& Johansen, 2014, Härkegård \& Glad, 2005: Johansen \& Fossen, 2013), as it can be used to select a subset of actuators (sensors) while optimising a cost function resulting in, for example, less power or fuel consumption, minimum actuator wear and tear, maintenance cost, etc. Equipped with a fault reconstruction framework (Casavola \& Garone, 2010, Edwards \& Tan, 2006, Tan \& Edwards, 2003; Zhang \& Jiang, 2002), it will be shown that the optimisation-based approach proposed in this paper for the design of row/column-sparse DOF controllers, by incorporating a secondary cost function into the main cost function used for optimal control design, can become a fault accommodation algorithm that searches for both a controller structure and a control law while minimising the performance degradation and allocation error.

In summary, the major focus of this paper is on the development of an approach for optimal actuator/sensor selection in over-actuated/sensed systems. However, this paper for dealing with this issue includes several novelties as follows:

- This paper develops a novel framework for the design of multi-channel $\mathscr{H}_{2}$ sparse row/column-wise DOF. To the authors' best knowledge, this is a new technology which can be exploited for the purpose of controller structure/control law co-design while employing only sensor's information.

- This paper proposes a novel scheme for the identification of favourable sparse row/column-wise patterns for DOF. It is worth noting that the current variable selection schemes typically amount to the selection of important individual variables; i.e. elements in the feedback gain, rather than the important groups of variables; i.e. rows/columns in the feedback gain. The contribution of this paper, in this regard, is to introduce a novel method that is able to penalise groups of variables (rows/columns in a matrix), so that a sparse row/column-wise (DOF) gain can be identified while minimising the performance degradation of the closed-loop system.

- A more practical result would be achieved by augmenting the optimisation problems exploited for the control synthesis by a set of LMI constraints to guarantee that the poles of the closed-loop system are located in a suitable subregion. Indeed, without having additional constraints to control the closed-loop transient behavior, a very sparse structure may be identified whilst an unsatisfactory time response and closed-loop damping may occur. This is a drawback of the existing literature for sparse control design that this paper aims to resolve. Moreover, as we take into consideration the mixed sparse multi-channel $\mathscr{H}_{2}$ DOF design problem (which can readily be extended to sparse multi-channel $\mathscr{H}_{2} / \mathscr{H}_{\infty}$ DOF design problem), a 
natural byproduct is to develop a form of pole clustering LMI characterisation which is applicable to our particular problem formulation. The contribution of this paper for this goal will extend the results in Chilali and Gahinet (1996) to more general LMI regions that can be combined with the specific LMI constraints developed for constraining the control performance.

- This approach has an immediate application in fault tolerant control (FTC) and specifically control allocation (CA) (Härkegård \& Glad, 2005 Johansen \& Fossen, 2013) for over-actuated/sensed systems. A distinct subsection is devoted to this issue.

Notation: $\left[\Phi_{i j}\right]_{r \times r}$ is a (block) matrix with (block) entries $\Phi_{i j}, i=1, \cdots, r, j=$ $1, \cdots, r$, and $\operatorname{diag}\left[\Phi_{i}\right]_{i=1}^{r}$ is a (block) diagonal matrix with (block) entries $\Phi_{i}, i=$ $1, \cdots, r$. Moreover, $\operatorname{col}\left(v_{i}(t)\right)_{i=1}^{r}$ denotes a (block) vector with (block) entries $v_{i}(t), i=$ $1, \cdots, r$. herm $(F)$, where $F$ is a square matrix, stands for $F+F^{*}$, and $*$ denotes Hermitian transpose. $\mathbb{C}$ denotes the complex plane and $\bar{z}$ denotes the conjugate of $z$.

\section{Problem Statement and Preliminaries}

\subsection{Problem statement}

Consider the following LTI system,

$$
\begin{aligned}
& \dot{x}(t)=A x(t)+B_{2} u(t)+B_{1} w(t) \\
& z(t)=C_{2} x(t)+D_{2} u(t) \\
& y(t)=C x(t),
\end{aligned}
$$

where $x \in \mathbb{R}^{n}, u \in \mathbb{R}^{m}, y \in \mathbb{R}^{p}, z \in \mathbb{R}^{q}$ and $w \in \mathbb{R}^{m_{w}}$ are the state vector, control input vector, output vector, $\mathscr{H}_{2}$ performance output vector, and the external disturbance of the system, respectively. The matrices in (1) are constant and of appropriate dimensions. It is also assumed that $\left(A, B_{2}\right)$ is stabilisable and $(A, C)$ is detectable. The main objective of this paper which is addressing the problem of optimal selection of the actuators and sensors can be stressed as the following problem:

Problem 1. Given a system with the state space representation in Equation (1), select a subset of available actuators/sensors and simultaneously find a controller that employs only the selected subset of actuators/sensors while minimising the degradation of an optimisation metric, say $\mathscr{H}_{2}$ norm of the closed-loop transfer function from $w$ to $z$, relative to the case where all the components are exploited.

To address Problem 1, we firstly construct a framework for the design of a controller which uses a priori specified subset of potential sensors and actuators. This framework can be employed to cope with different sensing and actuating capabilities. 
Problem 2. Given a system with the state space representation in Equation (1), find a controller employing a priori specified subset of potential actuators/sensors which minimises an optimisation metric, say $\mathscr{H}_{2}$ norm of the closed-loop transfer function from $w$ to $z$.

In order to deal with Problem 2 this paper tackles the DOF synthesis problem with multichannel $\mathscr{H}_{2}$ constraints as well as structural constraints. In what follows, to avoid the conservatism introduced by the so-called quadratic approach for the design of feedback gains with respect to multichannel $\mathscr{H}_{2}$ (or mixed $\mathscr{H}_{2}\left(\mathscr{H}_{\infty}\right)$ performance specifications, we develop a novel LMI method for the multichannel $\mathscr{H}_{2}$ DOF problem that can handle the requirements in Problem 2 Later in this paper, we will propose a method for addressing the issue of Problem 1 which can be regarded as optimal simultaneous actuator/sensor selection problem. Before doing so, let us present some definitions which we will make use of later in this paper.

Definition 1. A matrix is said to be a structure matrix if its elements are either 0 or 1. The structure matrix of a block matrix $Y=\left[Y_{i j}\right]_{m \times n}$ with $Y_{i j} \in \mathbb{R}^{r_{i} \times s_{j}}$ is $\mathrm{S}(Y) \triangleq\left[s_{i j}\right]_{m \times n}$ with

$$
s_{i j}= \begin{cases}0 & \text { if } Y_{i j}=0 \\ 1 & \text { otherwise } .\end{cases}
$$

Definition 2. Two matrices $Y_{1}$ and $Y_{2}$ are said to have the same structure if $\mathrm{S}\left(Y_{1}\right)=\mathrm{S}\left(Y_{2}\right)$.

Definition 3. The matrix $Y_{1}$ with $\mathrm{S}\left(Y_{1}\right) \triangleq\left[s_{i j}^{1}\right]_{m \times n}$ is said to be structurally a subset of $Y_{2}$ with $\mathrm{S}\left(Y_{2}\right) \triangleq\left[s_{i j}^{2}\right]_{m \times n}$ while $s_{i j}^{2}-s_{i j}^{1} \geq 0$. We denote this as $\mathrm{S}\left(Y_{1}\right) \subseteq \mathrm{S}\left(Y_{2}\right)$.

Definition 4. A matrix $\tilde{Y}$ is said to be sparse row-wise (column-wise) if its structure matrix $\tilde{\Gamma}$, i.e. $\mathrm{S}(\tilde{Y})=\tilde{\Gamma}$, includes (at least) one row (column) of all zeros.

\subsection{Controller dynamic}

We represent the DOF controller $\mathscr{K}(s)$ in state-space as:

$$
\begin{aligned}
\dot{x}_{c}(t) & =A_{c} x_{c}(t)+B_{c} y(t) \\
u(t) & =C_{c} x_{c}(t)+D_{c} y(t),
\end{aligned}
$$

where $x_{c} \in \mathbb{R}^{n}$ is the state vector of the controller and $A_{c}, B_{c}, C_{c}$ and $D_{c}$ are of appropriate dimensions. Let $\left(A_{c l}, B_{c l}, C_{c l}\right)$ denotes realisation of $T_{w z}:=C_{c l}(s I-$ $\left.A_{c l}\right)^{-1} B_{c l}$ (the closed loop transfer function from $w(t)$ to $z(t)$ ), where

$$
\begin{aligned}
A_{c l} & :=\left[\begin{array}{cc}
A+B_{2} D_{c} C & B_{2} C_{c} \\
B_{c} C & A_{c}
\end{array}\right], B_{c l}:=\left[\begin{array}{c}
B_{1} \\
0
\end{array}\right], \\
C_{c l} & :=\left[\begin{array}{ll}
C_{2}+D_{2} D_{c} C & D_{2} C_{c}
\end{array}\right] .
\end{aligned}
$$


Furthermore, as this paper aims to design the DOF subject to the constraints on the available sensors/actuators, we define $\mathrm{S}\left(\mathscr{K}_{a}\right) \subseteq \Gamma_{a}, \mathrm{~S}\left(\mathscr{K}_{S}\right) \subseteq \Gamma_{s}$, where $\Gamma_{a}$ and $\Gamma_{s}$ are a priori specified sparse row-wise and column-wise structures, respectively, and $\mathscr{K}_{a} \triangleq\left[\begin{array}{ll}C_{c} & D_{c}\end{array}\right], \mathscr{K}_{s} \triangleq\left[\begin{array}{ll}B_{c}^{T} & D_{c}^{T}\end{array}\right]^{T}$. It is not hard to realise that $\Gamma_{a}$ and $\Gamma_{s}$ can embody the pre-selected subsets of actuators and sensors, respectively. In other words, each nonzero row of $\mathscr{K}_{a}$ corresponds to an actuator used by the DOF controller, and likewise, each nonzero column of $\mathscr{K}_{s}$ corresponds to a sensor employed by the DOF controller, and thus, the number of nonzero rows (columns) of $\mathscr{K}_{a}\left(\mathscr{K}_{s}\right)$ is associated with the number of actuators (sensors).

We now provide a formulation for multichannel $\mathscr{H}_{2}$ DOF synthesis with the assumption of availability of all potential sensors or actuators. We later consider the problem of control synthesis subject to sparse row-wise and column-wise structure constraints from this full formulation.

\subsection{LMI characterisation}

\subsubsection{Structured $\mathscr{H}_{2}$ LMI characterisation for DOF synthesis}

Lemma 2.1. Let $\left(A_{c l}, B_{c l}, C_{c l}\right)$ denotes realisation of $T_{w z}$. The following three statements, involving $X>0, Z>0$, a general matrix variable $G$ are equivalent.

i) $A_{c l}$ is stable and $\left\|C_{c l}\left(s I-A_{c l}\right)^{-1} B_{c l}\right\|_{2}^{2}<\gamma$.

ii) $\exists X>0$ and $Z>0$ such that

$$
\begin{aligned}
& {\left[\begin{array}{cc}
A_{c l} X+X A_{c l}^{T} & \star \\
C_{c l} X & -\gamma I
\end{array}\right]<0,} \\
& {\left[\begin{array}{cc}
-Z & \star \\
B_{c l} & -X
\end{array}\right]<0,} \\
& \operatorname{trace}(Z)<1 .
\end{aligned}
$$

iii) $\exists X>0, Z>0$ and $G$ such that

$$
\begin{aligned}
& {\left[\begin{array}{ccc}
-\left(G+G^{T}\right) & \star & \star \\
A_{c l} G+X+G & -2 X & \star \\
C_{c l} G & 0 & -\gamma I
\end{array}\right]<0,} \\
& {\left[\begin{array}{cc}
-Z & \star \\
B_{c l} & -X
\end{array}\right]<0,} \\
& \operatorname{trace}(Z)<1 .
\end{aligned}
$$

Proof. Refer to the Appendix A.

Remark 1. The advantage of the item iii) over the LMI condition derived in Apkarian, Tuan, and Bernussou (2001) lies within the smaller dimension of the first LMI in iii) than that of in Apkarian et al. (2001). 
It should be emphasised that the specific LMI characterisation in (4) enables us to utilise different Lyapunov matrices $(X)$ for each of the involved LMI constraints in the problem. Moreover, the advantage of the LMI (4) lies within the fact that the product terms between the matrix $A_{c l}$ and the Lyapunov matrices have disappeared. More importantly, the control gain can be obtained independent of the Lyapunov matrix. This feature has a significant implication in the design of controllers satisfying multiple objectives such as the multi-channel $\mathscr{H}_{2}$ (or even mixed multi-channel $\mathscr{H}_{2} / \mathscr{H}_{\infty}$ ) specifications. It is also worth mentioning that, usually, the projection lemma (Gahinet \& Apkarian, 1993) is used, in the field of robust control, to eliminate the variable which contains the controller state-space data, and thus, deal only with a set of LMIs which include less number of decision variables. However, as can be seen, the proposed methodology here utilises the projection lemma in order to introduce an additional matrix variable $G$ to the problem.

\subsubsection{Multi-channel $\mathscr{H}_{2}$ synthesis using improved LMI characterisa- tions}

Our target is primarily to compute a full-order DOF $\mathscr{K}(s)$ presented in 2 which meets several performance specifications of the form

$$
\begin{array}{cl}
\text { minimise } & \left\|T_{w_{k} z_{k}}\right\|_{2} \\
\text { subject to }\left\|T_{w_{1} z_{1}}\right\|_{2}^{2}<\gamma_{1}, \cdots,\left\|T_{w_{k-1} z_{k-1}}\right\|_{2}^{2}<\gamma_{k-1}, \\
\left\|T_{w_{k+1} z_{k+1}}\right\|_{2}^{2}<\gamma_{k+1}, \cdots,\left\|T_{w_{\mathcal{N}} z_{\mathcal{N}}}\right\|_{2}^{2}<\gamma_{\mathcal{N}},
\end{array}
$$

where $\left\|T_{w_{i} z_{i}}\right\|_{2}:=\left\|L_{i} T_{w z} R_{i}\right\|_{2}$, in which $L_{i}$ and $R_{i}$ are selection matrices that determine the involved channels in the associated constraint. Hereafter in this paper, $\mathcal{N}$ denotes the number of channels. Furthermore, a realisation of the closed loop system $T_{w_{i} z_{i}}$ will be obtained by replacing matrices $B_{1}, C_{2}$ and $D_{2}$ by $B_{1, i}, C_{2, i}$ and $D_{2, i}, i=1, \cdots, \mathcal{N}$, respectively, in (1). Notice that, in such a case, the closed-loop performance is ensured by constraining (minimising) the $\mathscr{H}_{2}$ norm of the closed-loop transfer functions associated with the (input/output) signals $w_{i}=R_{i} w$ and $z_{i}=L_{i} z$; see De Oliveira, Gerome, and Bernussou (1999); Scherer, Gahinet, and Chilali (1997). Letting each channel to be associated with the LMI constraints in (4), (5), and (6), the desired characterisation with multi-channel specifications can be obtained by introducing a different Lyapunov variable for every channel and exploiting common variable $G$ for all channels. Hence, it is readily verified that using the part iii) of Lemma 2.1. the LMI characterisation 
for each channel $l$ can be written as:

$$
\begin{aligned}
& {\left[\begin{array}{ccc}
-\left(G+G^{T}\right) & \star & \star \\
A_{c l} G+X_{l}+G & -2 X_{l} & \star \\
C_{c l, l} G & 0 & -\gamma_{l} I
\end{array}\right]<0,} \\
& {\left[\begin{array}{cc}
-Z_{l} & \star \\
B_{c l, l} & -X_{l}
\end{array}\right]<0,} \\
& \operatorname{trace}\left(Z_{l}\right)<1,
\end{aligned}
$$

where $X_{l}>0, Z_{l}>0$ and $G$ are LMI variables. Now the multi-objective problem described in (7) can be set as

$$
\text { minimise } \gamma_{k}
$$

subject to (8), (9), and (10) for $k$-th channel,

(8), (9), and (10) for $l$-th channel, with given $\gamma_{l}, l=1, \cdots, \mathcal{N}, l \neq k$.

\subsubsection{Regional pole placement}

An adequate closed-loop transient response can be ensured by locating the closed-loop poles in a predetermined region (Chilali \& Gahinet, 1996). As the final goal of this paper is to optimise the number of actuators/sensors employed in the control loop, which is equivalent to row/column-wise sparsification of the feedback gain, without having additional constraints to control the closed-loop transient behavior, a very sparse structure might be identified, whereas an unsatisfactory time response and closed-loop damping may occur. As a result, the objective will be not only to sparsify the feedback gain while enforcing performance constraints of the closed-loop system, but also to guarantee that the poles of the closed-loop system are located in a suitable subregion.

In brief, an LMI region is a subset $\mathscr{D}$ of the complex plane as

$$
\mathscr{D}:=\left\{z \in \mathbb{C}: f_{\mathscr{D}}(z) \triangleq \Xi+z \Pi+\bar{z} \Pi^{T}<0\right\}
$$

in which $\Xi=\Xi^{T} \in \mathbb{R}^{\xi \times \xi}$ and $\Pi \in \mathbb{R}^{\xi \times \xi}$ are real matrices. $f_{\mathscr{D}}(z)$ is referred to as the characteristic equation of the region $\mathscr{D}$.

Definition 5 (Chilali, Gahinet, and Apkarian, 1999). A real matrix $\mathscr{A}$ is said to be $\mathscr{D}$-stable if all its eigenvalues lie within the LMI region $\mathscr{D}$.

Lemma 2.2 (Chilali et al. 1999). A real matrix $\mathscr{A}$ is $\mathscr{D}$-stable iff a symmetric matrix $X_{\mathscr{D}}>0$ exists such that

$$
\Xi \otimes X_{\mathscr{D}}+\Pi \otimes\left(X_{\mathscr{D}} \mathscr{A}\right)+\Pi^{T} \otimes\left(\mathscr{A}^{T} X_{\mathscr{D}}\right)<0,
$$

where $\otimes$ denotes the Kronecker product.

However, the regional pole placement problem considered in e.g. Chilali and Gahinet (1996) or Chilali et al. (1999) is developed for the conventional 
LMI characterisation of control problems such as $\mathscr{H}_{2}$ and/or $\mathscr{H}_{\infty}$. As discussed in Chilali et al. (1999), the synthesis problem is not convex when different Lyapunov matrices are used in the corresponding LMI region constraints. We alternatively extend the regional pole placement constraints in terms of the Lyapunov matrices $X_{l}, l=1, \cdots, \mathscr{M}(\mathscr{M}$ hereafter denotes the number of involved LMI region constraints) and the instrumental matrix $G$ to eliminate the product terms between the system matrix $A_{c l}$ and the Lyapunov matrix $X$. A sufficient condition is presented in the following theorem which is a suitable one for the control synthesis purposes.

Theorem 2.3. Let $\mathscr{A}, 0 \leq \Xi \in \mathbb{R}^{\xi \times \xi}$ and $\Pi \in \mathbb{R}^{\xi \times \xi}$ be real matrices. If there exist $G$ and symmetric positive definite matrix $X$ such that:

$$
\left[\begin{array}{ccc}
-I_{\xi} \otimes\left(G+G^{T}\right) & \star & \star \\
\Pi \otimes(\mathscr{A} G)+I_{\xi} \otimes X+I_{\xi} \otimes G & -2 I_{\xi} \otimes X & \star \\
\Xi^{\frac{1}{2}} \otimes G & 0 & -I_{\xi} \otimes X
\end{array}\right]<0,
$$

then $\mathscr{A}$ is $\mathscr{D}$-stable.

Proof. Refer to the Appendix B

This result is indeed a generalisation of the extended Lyapunov theorem presented in Theorem 3.1 of Apkarian et al. (2001). In other words, with $\boldsymbol{\Xi}=0$ and $\Pi=1$, (14) reduces to

$$
\left[\begin{array}{cc}
-\left(G+G^{T}\right) & \star \\
\mathscr{A} G+X+G & -2 X
\end{array}\right]<0,
$$

whose equivalence to the standard Lyapunov stability inequality for continuoustime LTI systems can be demonstrated through a similar manner presented in the proof of Lemma 2.1. As stated before, pole clustering specifications can be combined with other LMI performance constraints. For preserving tractability, the synthesis problem e.g. in Chilali and Gahinet (1996) requires to use the same matrix decision variable for all the LMIs involved in the problem, at the expense of additional conservatism. However, the extended LMI region developed here obviates the need for utilising the same Lyapunov variable in the synthesis problem. This also leads to the possibility to combine the pole clustering LMI regions to the aforementioned performance constraints in Section 2.3.2.

Specifically, we may aim to confine the closed-loop poles to the region $\mathbf{S}(\alpha, r, \theta)$ (see Chilali and Gahinet (1996)) which can ensure a minimum decay rate $\alpha$, a minimum damping ratio $\zeta=\cos \theta$, and a maximum undamped natural frequency $\omega_{d}=r \sin \theta$. The LMI region for an $\alpha$-stability, i.e. $\operatorname{Re}(z)<-\alpha$, can be obtained through (14, with

$$
\Xi=2 \alpha, \Pi=1 \text {. }
$$

Moreover, by letting

$$
\Xi=0, \Pi=\left[\begin{array}{cc}
\sin \theta & \cos \theta \\
-\cos \theta & \sin \theta
\end{array}\right],
$$


the LMI region for a conic sector $\mathbf{S}(0,0, \theta)$ is achieved. Furthermore, a disk centered at the origin with radius $r$ corresponds to

$$
\Xi=\left[\begin{array}{cc}
-r & 0 \\
0 & -r
\end{array}\right], \Pi=\left[\begin{array}{ll}
0 & 1 \\
0 & 0
\end{array}\right] .
$$

However, for this special pole clustering constraint, as $\Xi$ is not a semi-positive definite matrix, the LMI region cannot be obtained through (14). We can alternatively state the following theorem.

Theorem 2.4. Let $\mathscr{A}$ be a real matrix. The following statements are equivalent:

1) The eigenvalues of $\mathscr{A}$ lie in a disk centered at the origin with radius $\boldsymbol{r}$.

2) There exists a symmetric matrix $X>0$ such that

$$
\frac{1}{r} \mathscr{A} X \mathscr{A}^{T}-r X<0
$$

3) There exists a symmetric matrix $X>0$ such that

$$
\left[\begin{array}{cc}
-r X & \star \\
X \mathscr{A}^{T} & -r X
\end{array}\right]<0 .
$$

4) There exist a symmetric matrix $X>0$, and a matrix $G$ such that

$$
\left[\begin{array}{cc}
-r X & \star \\
G^{T} \mathscr{A}^{T} & -\left(G+G^{T}\right)+\frac{1}{r} X
\end{array}\right]<0 .
$$

Proof. Refer to the Appendix C.

Notice that the above theorem with $r=1$ reduces to the standard and extended Lyapunov stability inequality for discrete-time linear systems, respectively; e.g. see de Oliveira, Bernussou, and Geromel (1999).

Remark 2. Exploiting a common $\mathbf{G}$ may also lead to conservatism compared to the methods e.g. in Leite and Peres (2003). However, the methods in the aforementioned references are not beneficial for the control synthesis aims, unless gain scheduled controllers (Montagner $\&$ Peres, 2005) are considered. Moreover, by employing two instrumental variables, a different sufficient condition for robust D-stability has been developed in Peaucelle, Arzelier, Bachelier, and Bernussou (2000) which is not applicable to the continuous time control synthesis purposes. Nevertheless, the approach here can achieve less conservative results through employing non-common Lyapunov matrix variables for each design specification. 


\section{Row-Column-Sparse Multi-Channel $\mathscr{H}_{2}$ DOF With Regional Pole Placement}

Based on the discussions given in the previous section, we specify the requirements of Problem 2 in the following Problem.

Problem 3. Given a system with the state space representation in Equation (1), design a DOF such that it

- ensures the $\mathscr{H}_{2}$ performances with respect to channels $j=1, \cdots, \mathcal{N}, l \neq k$ which means that for a prescribed closed loop $\mathscr{H}_{2}$ performance $\gamma_{l}>0$, we have $\left\|T_{w_{l} z_{l}}\right\|_{2}^{2}<\gamma_{l}$;

- places the closed-loop poles in the predefined subregion;

- minimises the $\mathscr{H}_{2}$ performance of the $k$-th channel subject to the above constraints and $\mathrm{S}\left(\mathscr{K}_{a}\right) \subseteq \Gamma_{a}, \mathrm{~S}\left(\mathscr{K}_{s}\right) \subseteq \Gamma_{s}$, where $\Gamma_{a}$ and $\Gamma_{s}$ are given row and column sparse structures, respectively, and $\mathscr{K}_{a} \triangleq\left[\begin{array}{ll}C_{c} & D_{c}\end{array}\right], \mathscr{K}_{s} \triangleq\left[\begin{array}{ll}B_{c}^{T} & D_{c}^{T}\end{array}\right]^{T}$.

This problem can be formulated as an optimisation program in decision variables $X_{l}>0, Z_{l}>0, l=1, \cdots, \mathcal{N}+\mathscr{M}, G, A_{c}, B_{c}, C_{c}, D_{c}$ and $\gamma_{k}>0$ :

$$
\text { minimise } \gamma_{k}
$$

subject to (8), (9), and (10), for $k$-th channel,

(8), (9), and (10), for $l$-th channel, with given $\gamma_{l}, l=1, \cdots, \mathcal{N}, l \neq k$,

(14) and/or 21) (with $\mathscr{A}:=A_{c l}, X:=X_{l}, l=\mathcal{N}+1, \cdots, \mathcal{N}+\mathscr{M}$ ),

$\mathrm{S}\left(\mathscr{K}_{a}\right) \subseteq \Gamma_{a}$ and $\mathrm{S}\left(\mathscr{K}_{s}\right) \subseteq \Gamma_{s}$,

where $\Gamma_{a}$ and $\Gamma_{s}$ are preset row and column sparse structure matrices respectively. A difficulty in 22 is that it involves nonlinear terms $\left(A_{c l} G\right)$. The nonlinearities can be eliminated by some appropriate change of controller variables. In what follows, a change of variables is performed, inspired by the one introduced in Apkarian et al. (2001), to remove the nonlinear terms. We let the instrumental variable $G$ and $V=G^{-1}$ be of the form:

$$
G:=\left[\begin{array}{ll}
G_{1} & G_{3} \\
G_{2} & G_{4}
\end{array}\right], V:=\left[\begin{array}{ll}
V_{1} & V_{3} \\
V_{2} & V_{4}
\end{array}\right],
$$

where $\operatorname{dim} G_{1}=\operatorname{dim} V_{1}=\operatorname{dim} A$. As stated in e.g. Apkarian et al. (2001) and Chilali and Gahinet (1996), without loss of generality it can be assumed that $G_{2}$ and $V_{2}$ are invertible. Now consider the following invertible matrices:

$$
\mathrm{T}_{G}:=\left[\begin{array}{ll}
G_{1} & I \\
G_{2} & 0
\end{array}\right], \mathrm{T}_{V}:=\left[\begin{array}{ll}
I & V_{1} \\
0 & V_{2}
\end{array}\right] .
$$

It can readily be deduced that

$$
V \mathrm{\top}_{G}=\mathrm{\top}_{V}, G \mathrm{\top}_{V}=\mathrm{\top}_{G}
$$


Now the following theorem represents alternative tractable conditions which are useful for solving the optimisation problem 22 .

Theorem 3.1. Let $\mathscr{D}$ be an arbitrary LMI region with $(12)$ as its characteristic function. The sparse row-wise and column-wise multi-channel $\mathscr{H}_{2}$ DOF problem in (22) is solvable iff the following optimisation program has a solution in decision variables $\tilde{X}_{l}=\left[\begin{array}{ll}\tilde{X}_{l, 1} & \tilde{X}_{l, 2} \\ \tilde{X}_{l, 2}^{T} & \tilde{X}_{l, 3}\end{array}\right]>0, Z_{l}>0, l=1, \cdots, \mathcal{N}+\mathscr{M}, V_{1}, G_{1}, H, \tilde{A}_{c}$, $\tilde{B}_{c}, \tilde{C}_{c}, \tilde{D}_{c}$ and $\gamma_{k}>0$ :

$$
\text { minimise } \quad \gamma_{k}
$$

subject to (26), (27), and (10), for $k$-th channel,

(26), (27), and (10), for $l$-th channel, with given $\gamma_{l}, l=1, \cdots, \mathcal{N}, l \neq k$, (28) and/or 29) for $l=\mathscr{N}+1, \cdots, \mathcal{N}+\mathscr{M}$,

$$
\mathrm{S}\left(\tilde{\mathscr{K}}_{a}\right) \subseteq \Gamma_{a} \text { and } \mathrm{S}\left(\tilde{\mathscr{K}}_{s}\right) \subseteq \Gamma_{s} \text {, }
$$

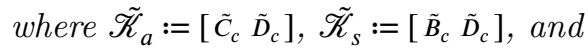

$$
\begin{gathered}
{\left[\begin{array}{ccccc}
-\left(G_{1}+G_{1}^{T}\right) & \star & \star & \star & \star \\
-H^{T}-I & -\left(V_{1}+V_{1}^{T}\right) & \star & \star & \star \\
A G_{1}+B_{2} \tilde{C}_{c}+\tilde{X}_{l, 1}+G_{1} & A+B_{2} \tilde{D}_{c} C+\tilde{X}_{l, 2}+I & -2 \tilde{X}_{l, 1} & \star & \star \\
\tilde{A}_{c}+\tilde{X}_{l, 2}^{T}+H^{T} & V_{1}^{T} A+\tilde{B}_{c} C+\tilde{X}_{l, 3}+V_{1}^{T} & -2 \tilde{X}_{l, 2}^{T} & -2 \tilde{X}_{l, 3} & \star \\
C_{2} G_{1}+D_{2} \tilde{C}_{c} & C_{2}+D_{2} \tilde{D}_{c} C & 0 & 0 & -\gamma_{l} I
\end{array}\right]<0} \\
\\
{\left[\begin{array}{ccc}
-Z & \star & \star \\
B_{1} & -\tilde{X}_{l, 1} & \star \\
V_{1}^{T} B_{1} & -\tilde{X}_{l, 2}^{T} & -\tilde{X}_{l, 3}
\end{array}\right]<0}
\end{gathered}
$$$$
\left[\begin{array}{ccc}
-I_{\xi} \otimes\left[\begin{array}{cc}
G_{1}+G_{1}^{T} & \star \\
H^{T}+I & V_{1}+V_{1}^{T}
\end{array}\right] \\
\Pi \otimes\left[\begin{array}{ccc}
A G_{1}+B_{2} \tilde{C}_{c} & A+B_{2} \tilde{D}_{C} C \\
\tilde{A}_{c} & V_{1}^{T} A+\tilde{B}_{c} C
\end{array}\right]+I_{\xi} \otimes\left[\begin{array}{cc}
\tilde{X}_{l, 1} & \star \\
\tilde{X}_{l, 2}^{T} & \tilde{X}_{l, 3}
\end{array}\right]+I_{\xi} \otimes\left[\begin{array}{cc}
G_{1} & I \\
H^{T} & V_{1}^{T}
\end{array}\right] & -2 I_{\xi} \otimes\left[\begin{array}{cc}
\tilde{X}_{l, 1} & \star \\
\tilde{X}_{l, 2}^{T} & \tilde{X}_{l, 3}
\end{array}\right] & \star \\
\Xi^{\frac{1}{2}} \otimes\left[\begin{array}{cc}
G_{1} & I \\
H^{T} & V_{1}^{T}
\end{array}\right] & 0 & -I_{\xi} \otimes\left[\begin{array}{cc}
\tilde{X}_{l, 1} & \star \\
\tilde{X}_{l, 2}^{T} & \tilde{X}_{l, 3}
\end{array}\right]
\end{array}\right]<0
$$

$$
\left[\begin{array}{cccc}
-r \tilde{X}_{l, 1} & \star & \star & \star \\
-r \tilde{X}_{l, 2}^{T} & -r \tilde{X}_{l, 3} & \star & \star \\
G_{1}^{T} A^{T}+\tilde{C}_{c}^{T} B_{2}^{T} & \tilde{A}_{c}^{T} & -\left(G_{1}+G_{1}^{T}\right)+\frac{1}{r} \tilde{X}_{l, 1} & \star \\
\left(A+B_{2} D_{c} C\right)^{T} & A^{T} V_{1}+C^{T} \tilde{B}_{c}^{T} & -H^{T}-I+\frac{1}{r} \tilde{X}_{l, 2}^{T} & -\left(V_{1}+V_{1}^{T}\right)+\frac{1}{r} \tilde{X}_{l, 3}
\end{array}\right]<0
$$

Proof. Performing congruence transformations $\operatorname{diag}\left(\mathrm{T}_{V}, \mathrm{~T}_{V}, I\right), \operatorname{diag}\left(I, \mathrm{~T}_{V}\right), \operatorname{diag}\left(I_{\xi} \otimes\right.$ $\left.\mathrm{T}_{V}, I_{\xi} \otimes \mathrm{T}_{V}, I_{\xi} \otimes \mathrm{T}_{V}\right)$, and $\operatorname{diag}\left(\mathrm{T}_{V}, \mathrm{~T}_{V}\right)$ in (8), (9), (14) and (21) respectively, along 
with the following change of variables:

$$
\begin{aligned}
\tilde{A}_{c} & :=V_{1}^{T} A G_{1}+V_{1}^{T} B_{2} D_{c} C G_{1}+V_{2}^{T} B_{c} C G_{1}+V_{1}^{T} B_{2} C_{c} G_{2}+V_{2}^{T} A_{c} G_{2}, \\
\tilde{B}_{c} & :=V_{1}^{T} B_{2} D_{c}+V_{2}^{T} B_{c}, \\
\tilde{C}_{c} & :=D_{c} C G_{1}+C_{c} G_{2}, \\
\tilde{D}_{c} & :=D_{c}, \\
\tilde{X}_{l} & :=\top_{V}^{T} X_{l} \top_{V}, \\
H & :=G_{1}^{T} V_{1}+G_{2}^{T} V_{2},
\end{aligned}
$$

lead to the LMIs in (26), (27), (28) and (29). Hence, the optimisation problem in $(22)$ is equivalent to optimisation problem in (25).

Remark 3. It is readily derived that

$$
\left[\begin{array}{cc}
\tilde{A}_{c} & \tilde{B}_{c} \\
\tilde{C}_{c} & \tilde{D}_{c}
\end{array}\right]=\left[\begin{array}{cc}
V_{2}^{T} & V_{1}^{T} B_{2} \\
0 & I
\end{array}\right]\left[\begin{array}{cc}
A_{c} & B_{c} \\
C_{c} & D_{c}
\end{array}\right]\left[\begin{array}{cc}
G_{2} & 0 \\
C G_{1} & I
\end{array}\right]+\left[\begin{array}{cc}
V_{1}^{T} A G_{1} & 0 \\
0 & 0
\end{array}\right] .
$$

As a result, it can be seen

$$
\tilde{\mathscr{K}}_{a}=\mathscr{K}_{a}\left[\begin{array}{cc}
G_{2} & 0 \\
C G_{1} & I
\end{array}\right] .
$$

Thus, since post-multiplication retains the row sparsity, it can be stated that $\mathscr{K}_{a}$ is row-sparse iff $\tilde{\mathscr{K}}_{a}$ is row-sparse. Moreover, as

$$
\tilde{\mathscr{K}}_{s}=\left[\begin{array}{cc}
V_{2}^{T} & V_{1}^{T} B_{2} \\
0 & I
\end{array}\right] \mathscr{K}_{s},
$$

and since pre-multiplication retains the column sparsity, then $\mathscr{K}_{s}$ is columnsparse iff $\tilde{\mathscr{K}}_{s}$ is column sparse.

Obtaining a solution from (25), the DOF controller can readily be found from:

1) computing a full rank factorisation $G_{2}^{T} V_{2}$ of $H-G_{1}^{T} V_{1}$ and thus invertible $G_{2}$ and $V_{2}$;

2 ) solving the equations 300 - 33 for controller matrices $A_{c}, B_{c}, C_{c}$ and $D_{c}$.

\section{Optimal Actuator/Sensor Selection Problem}

While the previous section considered the design of a multi-channel $\mathscr{H}_{2} \mathrm{DOF}$ with a priori specified set of actuators and sensors, this section aims to address the objective mentioned in Problem 1, which is exploring favourable row and column sparse DOF gains (selection of actuators and sensors). To do so, an optimisation framework is proposed here, by incorporating the column-wise sparsity of $\tilde{\mathscr{K}}_{s}$ and row-wise sparsity of $\tilde{\mathscr{K}}_{a}$ into the index function, which is encapsulated in the following problem. 
Problem 4. Given a system with the state space representation in Equation (1), find $\tilde{X}_{l}=\left[\begin{array}{cc}\tilde{X}_{l, 1} & \tilde{X}_{l, 2} \\ \tilde{X}_{l, 2}^{T} & \tilde{X}_{l, 3}\end{array}\right]>0, Z_{l}>0, l=1, \cdots, \mathcal{N}+\mathscr{M}, V_{1}, G_{1}, H, \tilde{A}_{c}, \tilde{B}_{c}, \tilde{C}_{c}, \tilde{D}_{c}$ and $\gamma_{k}>0$ in the following optimisation program:

$$
\text { minimise } \gamma_{k}+\eta_{a}\left\|\tilde{\mathscr{K}}_{a}\right\|_{\text {row- } \ell_{0}}+\eta_{s}\left\|\tilde{\mathscr{K}}_{s}\right\|_{\text {col- } \ell_{0}},
$$

subject to the constraints given in 25), excluding the structural constraints on $\tilde{\mathscr{K}}_{a}$ and $\tilde{\mathscr{K}}_{s}$, where the row- $\ell_{0}\left(\right.$ col- $\left.\hat{\ell}_{0}\right)$ is a quasi-norm that counts the number of non-zero rows (columns) of $\tilde{\mathscr{K}}_{a}\left(\tilde{\mathscr{K}}_{s}\right)$, and $\eta_{a}>0\left(\eta_{s}>0\right)$ is the regularisation parameter that implies the emphasis on the row-sparsity (column-sparsity) of $\tilde{\mathscr{K}}_{a}\left(\tilde{\mathscr{K}}_{s}\right)$; i.e. a larger $\eta_{a}\left(\eta_{s}\right)$ will result in a more row-sparse (column-sparse) $\tilde{\mathscr{K}}_{a}\left(\tilde{\mathscr{K}}_{s}\right)$.

Clearly 35 is a combinatorial problem and broadly speaking impossible to solve. This is because an intractable combinatorial search is required to address this problem whose computation time grows faster than polynomial, as the order of the system under study grows (Schuler et al. 2014). In the literature, several alternatives such as $\ell_{1}$-norm or weighted $\ell_{1}$-norm are proposed as convex relaxations of the $\ell_{0}$-quasi-norm (Candes, Wakin, \& Boyd, 2008). The so-called reweighted $\ell_{1}$ (REL1) minimisation method (Candes et al., 2008) is an iterative scheme, in which at each iteration a convex optimisation problem is solved by employing weighted $\ell_{1}$-norm rather than the quasi $\ell_{0}$-norm, whilst the weights are updated inversely proportional to the previous iteration's solution. The REL1 algorithm has recently been used by a number of researchers (e.g. see Fardad and Jovanovic (2014), Zoltowski, Dhingra, Lin, and Jovanovic (2014)) for the design of sparse controllers for the distributed systems. Notice that the variable selection in the aforementioned papers typically amounts to the selection of important individual variables (elements in the feedback gain) rather than the important groups of variables (rows or columns). The contribution of this paper, in this regard, is to introduce a novel method that is able to penalise groups of variables (rows or columns in a matrix), so that a sparse row/column-wise DOF controller can be identified while minimising the performance degradation of the closed-loop system. Let us now relax Problem 4 as follows:

Problem 5. Given a system with the state space representation in Equation (1), find $\tilde{X}_{l}=\left[\begin{array}{cc}\tilde{X}_{l, 1} & \tilde{X}_{l, 2} \\ \tilde{X}_{l, 2}^{T} & \tilde{X}_{l, 3}\end{array}\right]>0, Z_{l}>0, l=1, \cdots, \mathscr{N}+\mathscr{M}, V_{1}, G_{1}, H, \tilde{A}_{c}, \tilde{B}_{c}, \tilde{C}_{c}, \tilde{D}_{c}$ and $\gamma_{k}>0$ in the following convex optimisation program:

$$
\text { minimise } \gamma_{k}+\eta_{a} f\left(\tilde{\mathscr{K}}_{a}\right)+\eta_{s} g\left(\tilde{\mathscr{K}}_{s}\right) \text {, }
$$

subject to the constraints given in 25), excluding the structural constraints on $\tilde{\mathscr{K}}_{a}$ and $\tilde{\mathscr{K}}_{s}$, where $f(\cdot)(g(\cdot))$ denotes the relaxed row-sparsity (column-sparsity) promoting function.

Different choices for relaxed row/column-sparsity promoting function will be proposed in the following of this section. 


\subsection{Row (column) sparsity promoting penalty function}

Consider the row-sparsity promoting function as

$$
f\left(\tilde{\mathscr{K}}_{a}\right)=\sum_{i, j} W_{a, i}\left|\tilde{\mathscr{K}}_{a, i j}\right|
$$

where $W_{a, i}$ denotes the weight corresponds to the $i$-th row of $\tilde{\mathscr{K}}_{a}$. One may now resort to update the weights inversely proportional to the $\ell_{1}$-norm of its corresponding row in $\tilde{\mathscr{K}}_{a}$ obtained at the previous iteration as:

$$
W_{a, i}^{h}=\frac{1}{\sum_{j}\left|\tilde{\mathscr{K}}_{a, i j}^{(h-1)}\right|+\epsilon},
$$

where $h$ denotes the current iteration and $0<\epsilon \ll 1$ is used to provide stability, and form the weighting matrix as $W_{a}=\operatorname{diag}\left[W_{a, i}\right]_{i=1}^{m}$. One can also imagine a variety of possible norms in place of $(38)$, e.g. $\ell_{2}$-norm and $\ell_{\infty}$-norm. Therefore the update rule in 38 can be revised to:

$$
W_{a, i}^{h}=\frac{1}{\sqrt{\sum_{j}\left|\tilde{\mathscr{K}}_{a, i j}^{(h-1)}\right|^{2}}+\epsilon},
$$

for $\ell_{2}$-norm, and

$$
W_{a, i}^{h}=\frac{1}{\max _{j}\left(\left|\tilde{\mathscr{K}}_{a, i j}^{(h-1)}\right|\right)+\epsilon}
$$

for $\ell_{\infty}$-norm. Although we have found the one in 38 performs well in a wide range of experiments, using $\ell_{2}$-norm or $\ell_{\infty}$-norm can sometimes outperform $\ell_{1}$-norm. Notice that these norms only indicate the method which allows us to update the weighting matrix $W_{a}$, while the sparsity term in the objective function is considered as the one in (37).

Remark 4. Instead of using (37) which is the weighted $\ell_{1}$-norm of $\ell_{1}$-norms of the rows of $\tilde{\mathscr{K}}_{a}$, one can use

$$
f\left(\tilde{\mathscr{K}}_{a}\right)=\left(\sum_{i, j} W_{a, i}^{2}\left|\tilde{\mathscr{K}}_{a, i j}\right|^{2}\right)^{\frac{1}{2}} .
$$

It is not hard to comprehend that (41) denotes the weighted Frobenius-norm of $\tilde{\mathscr{K}}_{a}$. As discussed in Candes et al. (2008), while unweighted $\ell_{2}$-norm minimisation does not encourage sparsity, its reweighted algorithm can be used as a (row or column)-sparsity promoting function.

For the sensor selection problem, we can similarly replace column- $\ell_{0}$ by a relaxed column-sparsity promoting function $\left(g\left(\tilde{\mathscr{K}}_{s}\right)\right)$ for which different choices can be considered, which are basically the counterparts of the ones given for 
the actuator selection problem. For instance, the column-sparsity promoting function can be considered as:

$$
g\left(\tilde{\mathscr{K}}_{s}\right)=\sum_{i, j}\left|\tilde{\mathscr{K}}_{s, i j}\right| W_{s, j},
$$

while the update rule e.g. in 38 can be converted to:

$$
W_{s, j}^{h}=\frac{1}{\sum_{i}\left|\tilde{\mathscr{K}}_{s, i j}^{(h-1)}\right|+\epsilon}
$$

and the weighting matrix is similarly formed as $W_{s}=\operatorname{diag}\left[W_{s, j}\right]_{j=1}^{p}$. An iterative scheme for identifying a row-sparse $\tilde{\mathscr{K}}_{a}$ and a column-sparse $\tilde{\mathscr{K}}_{s}$ is proposed in the next subsection.

\subsection{Identification of row/column sparse patterns through DOF}

Define the matrices $R_{a}=\left[0_{(n+p) \times n} 1_{(n+p) \times m}\right]$ and $R_{s}=\left[0_{(n+m) \times n} 1_{(n+m) \times p}\right]^{T}$. The optimisation problem in (36), by letting $f\left(\tilde{\mathscr{K}}_{a}\right)$ and $g\left(\tilde{\mathscr{K}}_{s}\right)$ as 37 and 42 respectively, is equivalent to

$$
\text { minimise } \gamma_{k}+\eta_{a} \operatorname{trace}\left(\boldsymbol{R}_{a} \mathscr{W}\right)+\eta_{s} \operatorname{trace}\left(\mathscr{W} \boldsymbol{R}_{s}\right)
$$

subject to (26), (27), and (10), for $k$-th channel,

(26), (27), and (10), for $l$-th channel, with given $\gamma_{l}, l \neq k, l=1, \cdots, \mathcal{N}$, (28) and/or 29 for $l=\mathscr{N}+1, \cdots, \mathcal{N}+\mathscr{M}$,

$-\mathscr{W} \leq \operatorname{diag}\left(I, W_{a}\right) \cdot \tilde{\mathscr{K}} \cdot \operatorname{diag}\left(I, W_{s}\right) \leq \mathscr{W}$,

where $W_{a}$ and $W_{s}$ denote the weighting matrices and the last inequality is element-wise with $\mathscr{W} \in \mathbb{R}^{(n+m) \times(n+p)}$ whose entries are nonnegative. Besides, to solve the above optimisation problem, the following algorithm is utilised:

Algorithm 1. 1) With given $\epsilon>0, \kappa>0, \eta_{a}>0$ and $\eta_{s}>0$, initialise $W_{a}=$ $I_{m}, W_{s}=I_{p}, h=1$ and $\tilde{\mathscr{K}}^{h}=0$.

2) Solve the minimisation problem (44) to obtain $\tilde{\mathscr{K}}^{\star}$ and the associated $\tilde{\mathscr{K}}_{a}^{\star}=\left[\begin{array}{cc}\tilde{C}_{c}^{\star} & \tilde{D}_{c}^{\star}\end{array}\right]$ and $\tilde{\mathscr{K}}_{s}^{\star}=\left[\begin{array}{cc}\tilde{B}_{c}^{\star} & \tilde{D}_{c}^{\star}\end{array}\right]^{T}$.

3) Update $W_{a, i}^{h}$ using the update rule in (38) (or (39) or (40)) and its counterpart $W_{s, i}^{h}$ for sensor selection e.g. in (43), form $W_{a}^{h}=\operatorname{diag}\left[W_{a, i}^{h}\right]_{i=1}^{m}$ and $W_{s}^{h}=\operatorname{diag}\left[W_{s, j}^{h}\right]_{j=1}^{p}$.

5) If $\left\|\tilde{\mathscr{K}}^{\star}-\tilde{\mathscr{K}}^{h}\right\| \leq \kappa$ go to Step 6 , else $\tilde{\mathscr{K}}^{h}=\tilde{\mathscr{K}}^{\star}, h=h+1$ and return to Step 2.

6) Let the unnecessary rows of $\tilde{\mathscr{K}}_{a}^{\star}$ and columns of $\tilde{\mathscr{K}}_{s}^{\star}$ be zero and return $\Gamma_{a}^{\star}=\mathrm{S}\left(\tilde{\mathscr{K}}_{a}^{\star}\right)$ and $\Gamma_{s}^{\star}=\mathrm{S}\left(\tilde{\mathscr{K}}_{s}^{\star}\right)$. 
We denote the obtained structure of the minimisation problem 44, as $\mathrm{S}\left(\tilde{\mathscr{K}}_{a}\right) \triangleq \Gamma_{a}$ and $\mathrm{S}\left(\tilde{\mathscr{K}}_{S}\right) \triangleq \Gamma_{s}$. Eventually, in order to find the multi-channel $\mathscr{H}_{2}$ DOF associated with the achieved $\Gamma_{a}$ and $\Gamma_{s}$, we turn to the minimisation problem in 25).

\subsection{Application to fault accommodation for over-actuated/over- sensed systems}

Let us rewrite the system in (1) for the cases that are subject to actuator/sensor faults or failures:

$$
\begin{aligned}
\dot{x}(t) & =A x(t)+\tilde{B}_{2}(t) u(t)+B_{1} w(t) \\
z(t) & =\tilde{C}_{z}(t) x(t)+\tilde{D}_{z}(t) u(t) \\
y(t) & =\tilde{C}(t) x(t)
\end{aligned}
$$

where $\tilde{B}_{2}(t)=B_{2} \Phi_{u}(t), \tilde{D}_{z}(t)=D_{2} \Phi_{u}^{-1}(t)$, in which $\Phi_{u}(t)=\operatorname{diag}\left(\phi_{u, 1}(t), \cdots, \phi_{u, m}(t)\right)$, whereas $\mathscr{K}_{u}(t)=\operatorname{diag}\left(k_{u, 1}(t), \cdots, k_{u, m}(t)\right) \triangleq I_{m}-\Phi_{u}(t)$ is referred to as the effectiveness gain (Alwi \& Edwards, 2010, Casavola \& Garone, 2010) with $k_{u, i}(t)$ denoting some scalars satisfying $0 \leq k_{u, i}(t) \leq 1$; i.e. $k_{u, i}(t)=0$ implies that the $i$-th actuator is fault-free, whilst $0<k_{u, i}(t)<1$ represents a fault in the $i$-th actuator, and $k_{u, i}(t)=1$ shows the actuator failure. Similarly, $\tilde{C}(t)=\Phi_{y}(t) C, \tilde{C}_{z}(t)=C_{z} \Phi_{y}^{-1}(t) C$, in which $C_{z} \in \mathbb{R}^{q \times p}, \Phi_{y}(t)=\operatorname{diag}\left(\phi_{y, 1}(t), \cdots, \phi_{y, p}(t)\right)$, with $\phi_{y, j}(t)$ denoting some scalars satisfying $0 \leq \phi_{y, j}(t) \leq 1$; i.e. $\phi_{y, j}(t)=1$ implies that the $j$-th sensor is fault-free, whilst $0<\phi_{y, j}(t)<1$ represents a fault in the $j$-th sensor, and $\phi_{y, j}(t)=0$ demonstrates the sensor failure.

Assume the system 45 involves multiplicative faults. Employing an actuator fault reconstruction framework such as the one in Tan and Edwards (2003) or Kalman filter-based fault reconstruction frameworks (Zhang \& Jiang, 2002), and a sensor fault reconstruction framework such as the scheme proposed in Edwards and $\operatorname{Tan}(2006), \Phi_{u}(t)$ and $\Phi_{y}(t)$ can be computed in a real-time manner. Now, the optimisation problem in 25 , excluded from the structural constraints, by replacing $\boldsymbol{B}_{2}, \boldsymbol{C}_{2}$ and $\boldsymbol{D}_{2}$, with $\widetilde{\boldsymbol{B}}_{2}(t), \tilde{\boldsymbol{C}}_{z}(t)$ and $\tilde{D}_{z}(t)$, respectively, can be considered as a sensor/actuator fault accommodation algorithm that searches for a control law in faulty situation while minimising the performance degradation. When no fault exists, $\Phi_{u}=I_{m}$ and $\Phi_{y}=I_{p}$. On the other hand, it is obvious that if $\phi_{u, i} \rightarrow 0$ and/or $\phi_{y, i} \rightarrow 0$, then $\phi_{u, i}^{-1} \rightarrow \infty$ and/or $\phi_{y, i}^{-1} \rightarrow \infty$, respectively, and hence, the associated components $u_{i}$ and/or $y_{i}$ are weighted heavily in the $\mathscr{H}_{2}$ performance output.

As discussed earlier, in over-actuated systems, in order to, for example, minimise power or fuel consumption and/or actuator wear and tear, one may resort to select a subset of available actuators (Johansen \& Fossen, 2013). The same statement can be mentioned for over-sensed systems. Thus, roughly speaking, a subset with small number of actuators/sensors is often preferred to a large subset. This issue is of importance in fault-tolerance, as redundancy of actuators and/or sensors may be desired. In other words, the component selection 
problem can be used to select subsets that show no redundancy. As explained previously, the optimisation problem proposed in (35) (or its relaxed version in (36)), achieved by incorporating two secondary cost functions into the main cost function, aims to select a subset of available actuators/sensors in an optimal manner. It is not hard to realise that the optimisation problem in (36), by replacing $B_{2}, C_{2}$, and $D_{2}$, with $\tilde{B}_{2}(t), \tilde{C}_{z}(t)$, and $\tilde{D}_{z}(t)$, respectively, can specifically be employed as a simultaneous sensor/actuator fault tolerant control algorithm that searches for a sparse control law while minimising the performance degradation and allocation error. In summary, undesirable actuators/sensors would be neglected due to i) the high execution cost (identified by solving the optimisation problem (36) or ii) failure (identified by a fault reconstruction framework) by means of penalising the associated components in the control/output signals by large weights, i.e. $\phi_{u, i}^{-1} \rightarrow \infty$ and/or $\phi_{y, i}^{-1} \rightarrow \infty$.

\section{$5 \quad$ Numerical examples}

Two numerical examples are presented here in order to evaluate the effectiveness of the proposed method for the problem of actuator-sensor selection via DOF approach. All the LMI optimisation problems are solved by YALMIP (Löfberg, 2004) as the interface and SDPT3 (Toh, Todd, Tütüncü, \& Tutuncu, 1998) as the solver.

\subsection{Example 1}

Let us consider the problem HE3 from COMPleib (Leibfritz \& Lipinski, 2003). This problem is related to the eight order linearised state space model of the dynamics of the Bell201A-1 helicopter that has four inputs and six outputs. The system matrices can be seen in Leibfritz and Lipinski (2003). Besides, we let $C_{1}=\left[\begin{array}{c}C \\ 0_{4 \times 8}\end{array}\right], D_{1}=\left[\begin{array}{c}0_{6 \times 4} \\ I_{4}\end{array}\right], C_{2}=\left[\begin{array}{c}\tilde{C} \\ 0_{4 \times 8}\end{array}\right]$ and $D_{2}=\left[\begin{array}{c}0_{2 \times 4} \\ I_{4}\end{array}\right]$, where

$$
\tilde{C}=\left[\begin{array}{llllllll}
1 & 0 & 0 & 0 & 0 & 0 & 0 & 0 \\
0 & 0 & 0 & 1 & 0 & 0 & 0 & 0
\end{array}\right]
$$

The objective is first to identify the minimum number of actuators and sensors by which the closed-loop stability is not violated, the closed-loop damping is satisfactory, i.e. $\zeta>0.8660$, and its decay rate is limited by $x<-\alpha<-0.2$, and the performance degradation of the closed-loop system is restricted. Solving the optimisation problem in 25 , with $\mathcal{N}=2, \gamma_{2}<10, \Gamma_{a}=\mathbb{1}_{m \times(n+p)}, \Gamma_{s}=\mathbb{1}_{(n+m) \times p}$, involved with the LMI regional pole placement constraints associated with the above pole clustering constraints, results in a true $\mathrm{H}_{2}$ cost of 0.8118 for channel 1 and 0.4585 for channel 2 .

Remark 5. Note that the value of the $\mathscr{H}_{2}$ cost obtained from 25) is not the true one, due to the conservatism introduced by employing common $G, A_{c}, B_{c}$, $C_{c}$ and $D_{c}$. Nevertheless, the true value can be computed by solving the following 
Lyapunov equation

$$
X_{i, \text { true }} A_{c l}+A_{c l}^{T} X_{i, t r u e}+C_{c l, i}^{T} C_{c l, i}=0 .
$$

One can then find the $\mathscr{H}_{2}$ cost as $\sqrt{\operatorname{trace}\left(B_{c l, i}^{T} X_{i, t r u e} B_{c l, i}\right)}$.

Now we solve the optimisation problem in (36) by utilising Algorithm 1 with $\kappa=0.1, \epsilon=0.0001$ and exploiting the row-sparsity promoting function (37) as well as the column-sparsity promoting function (42), in addition to the update rule in (39) and its counterpart for the sensor selection, respectively. As increases $\eta_{a}$ and $\eta_{s}$, the underlying $\mathscr{K}_{a}$ and $\mathscr{K}_{s}$ gradually become row-sparse and column-sparse, respectively. For example with $\eta_{a}=0.1$ and $\eta_{s}=0.5$, the algorithm suggests excluding the control system from the sensors 4 and 5 . We then solve the optimisation problem in 25 with the obtained pattern for $\Gamma_{s}$ ( $\Gamma_{a}$ is still non-structured), for the design of DOF controller. In such a case, no degradation occurs in the upper bound of $\boldsymbol{H}_{2}$-norm of channels 1 and 2 .

Further with $\eta_{a}=1$ and $\eta_{s}=0.5$, the algorithm suggests exploiting only the control inputs 2 and 4 while the controller does not receive information of sensors 4 and 5 , but at the expense of about $25 \% \mathrm{H}_{2}$ performance degradation in channel 1 compared to the non-sparse $\mathscr{K}$.

Moreover, letting $\eta_{a}=2$ and $\eta_{s}=10$, Algorithm 1 proposes exploiting sensors 1, 2 and 3 and actuators 2 and $4\left(\left\|\mathscr{K}_{a}\right\|_{\text {row }-\ell_{0}}=2\right.$ and $\left.\left\|\mathscr{K}_{s}\right\|_{\text {col }-\ell_{0}}=3\right)$. Again, about $25 \% \mathrm{H}_{2}$ performance degradation happens in channel 1. As can be seen, the performance degradation that occurs, in this case, is the same as the previous case with $\left\|\mathscr{K}_{a}\right\|_{\text {row }-\ell_{0}}=2$ and $\left\|\mathscr{K}_{s}\right\|_{\text {col }-\ell_{0}}=4$. The closed-loop eigenvalues are:

$$
\begin{aligned}
& \{-11.3706,-1.8788 \pm 0.2658 i,-1.1343 \pm 0.2956 i, \\
& \quad-1.0941 \pm 0.1427 i,-0.3859 \pm 0.5176 i,-0.2412 \pm 0.3487 i, \\
& -0.6508 \pm 0.3724 i,-0.8371,-0.2329 \pm 0.0368 i\},
\end{aligned}
$$

that all of them lie within the preselected region.

\subsection{Example 2: Flight control}

Now we consider the B747 aircraft (Alwi \& Edwards, 2010) whose 12 rigid body states can be split into two separate axes: 6 longitudinal axis states and 6 lateral and directional axes states. The same as in Alwi and Edwards (2010) we only consider the first four states of the lateral axis which are the roll rate $p$, yaw rate $r$, sideslip angle $\beta$, and roll angle $\phi$. Considering an operating condition of $263,000 \mathrm{Kg}, 92.6 \mathrm{~m} / \mathrm{s}$ true airspeed, and $600 \mathrm{~m}$ altitude at $25.6 \%$ of maximum thrust and at a $20^{\circ}$ flap position, a linear model can be obtained. In this case, the lateral system, about the trim condition, can be represented as:

$$
A=\left[\begin{array}{cccc}
-1.0579 & 0.1718 & -1.6478 & 0.0004 \\
-0.1186 & -0.2066 & 0.2767 & -0.0019 \\
0.1014 & -0.9887 & -0.0999 & 0.1055 \\
1.0000 & 0.0893 & 0 & 0
\end{array}\right],
$$




$$
B_{2}=\left[\begin{array}{ccccccc}
-0.0832 & 0.0832 & -0.2285 & 0.2285 & -0.2625 & -0.0678 & 0.0678 \\
-0.0154 & 0.0154 & -0.0123 & 0.0123 & -0.0180 & -0.0052 & 0.0052 \\
0 & 0 & 0 & 0 & 0.0017 & 0.0006 & -0.0006 \\
0 & 0 & 0 & 0 & 0 & 0 & 0 \\
& 0.2625 & 0.1187 & 0.0246 & 0.0140 & -0.0140 & -0.0246 \\
& 0.0180 & -0.2478 & 0.1269 & 0.0724 & -0.0724 & -0.1269 \\
& -0.0017 & 0.0174 & 0.0005 & 0.0005 & -0.0005 & -0.0005
\end{array}\right]
$$

Note that the lateral control surfaces are

$\delta_{\text {lat }}=\left[\begin{array}{lllllllllllll}\delta_{\text {air }} & \delta_{\text {ail }} & \delta_{a o r} & \delta_{a o l} & \delta_{s p 1-4} & \delta_{s p 5} & \delta_{s p 8} & \delta_{s p 9-12} & \delta_{r} & e_{1} & e_{2} & e_{3} & e_{4}\end{array}\right]$,

denoting aileron deflection (right and left - inner and outer)(rad), spoiler deflections (left: 1-4 and 5, right: 8 and 9-12) (rad), rudder deflection (rad) and lateral contributions to the engine pressure ratios (EPR), respectively. We let the system outputs be sideslip angle $\beta$ and roll angle $\phi$, and thus the output distribution matrix is

$$
C_{l}=\left[\begin{array}{llll}
0 & 0 & 1 & 0 \\
0 & 0 & 0 & 1
\end{array}\right]
$$

Exploiting an integral action, we include a tracking facility in the problem. Defining

$$
\dot{\vartheta}(t)=r(t)-y_{l}(t)
$$

where $\mathrm{r}(t)$ is the input reference to be tracked by $y_{l}(t)=C_{l} x(t)$, with $C_{l} \in \mathbb{R}^{l \times n}$, and $\vartheta$ represents the integral of the tracking error, i.e. $\mathrm{r}(t)-y_{l}(t)$, and introducing $\tilde{x}:=\left[\begin{array}{l}x \\ 9\end{array}\right]$, an augmented system can be derived as:

$$
\begin{aligned}
& \dot{\tilde{x}}(t)=\tilde{A} \tilde{x}(t)+\tilde{B} u(t)+\tilde{B}_{1} w(t)+B_{\mathrm{r}} \mathrm{r}(t) \\
& \tilde{y}(t)=\tilde{C} \tilde{x}(t), \\
& \tilde{z}(t)=\tilde{C}_{2} \tilde{x}(t)+\tilde{D}_{2} u(t),
\end{aligned}
$$

with

$$
\begin{gathered}
\tilde{A}=\left[\begin{array}{cc}
A & 0 \\
-C_{l} & 0
\end{array}\right], \tilde{B}=\left[\begin{array}{c}
B_{2} \\
0
\end{array}\right], B_{\mathrm{r}}=\left[\begin{array}{l}
0 \\
I_{l}
\end{array}\right], \tilde{C}=\left[\begin{array}{cc}
I_{n} & 0 \\
0 & C_{l}
\end{array}\right], \tilde{B}_{1}=\left[\begin{array}{c}
B_{1} \\
0
\end{array}\right]=\left[\begin{array}{c}
I_{4} \\
0_{2 \times 4}
\end{array}\right], \\
\tilde{C}_{2}=\left[\begin{array}{c}
\operatorname{diag}(50,50,10,10,20,20) \\
0_{13 \times 6}
\end{array}\right], \tilde{D}_{2}=\left[\begin{array}{c}
0_{6 \times 13} \\
\operatorname{diag}\left(\frac{\sqrt{2}}{2} I_{8}, \sqrt{2} I_{5}\right)
\end{array}\right], l=2, n=4, m=13 .
\end{gathered}
$$

Note that the last two nonzero terms of $\tilde{C}_{2}$ are associated with the integral action. Further, the first and second terms of $\tilde{C}_{2}$ are more heavily weighted in comparison with the third and fourth terms to provide an adequate quick closedloop response in terms of the angular acceleration in roll and yaw. Note also 


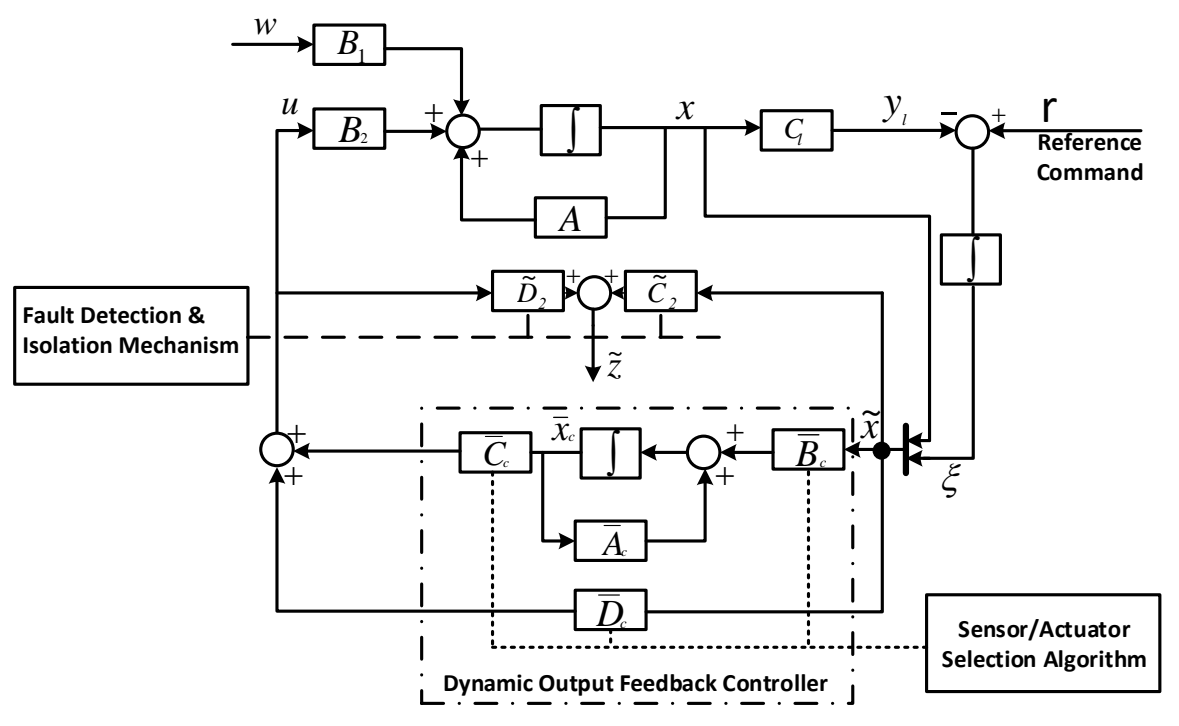

Figure 1: Schematic of the proposed reference tracking DOF controller

that if the matrix pair $\left(A, B_{2}\right)$ is controllable and the matrix triplet $\left(A, B_{2}, C_{l}\right)$ has no zeros at the origin, it can be shown that $(\tilde{A}, \tilde{B})$ is controllable $($ Alwi \& Edwards, 2010). Now for the reference output tracking control objective, the full order $\overline{\mathrm{DOF}}$ is constructed as:

$$
\left[\begin{array}{c}
\dot{\bar{x}}_{c}(t) \\
u(t)
\end{array}\right]=\left[\begin{array}{cc}
\bar{A}_{c} & \bar{B}_{c} \\
\bar{C}_{c} & \bar{D}_{c}
\end{array}\right]\left[\begin{array}{c}
\bar{x}_{c}(t) \\
\tilde{x}(t)
\end{array}\right]
$$

where $\tilde{x} \in \mathbb{R}^{n+l}$ is the reference output tracking controller state and $\bar{A}_{c} \in \mathbb{R}^{(n+l) \times(n+l)}$. The schematic of the proposed reference tracking DOF controller is shown in Figure 1. We also aim to assign the closed-loop poles within a subregion achieved by the intersection of the half-plane $x<-\alpha<-0.1$, and the sector centred at the origin making an angle of $\theta=\pi / 3$. By imposing no structure on $\tilde{\mathscr{K}}_{a}$ and $\tilde{\mathscr{K}}_{s}$; i.e. $\Gamma_{a}=\mathbb{1}_{m \times[2(n+l)]}$ and $\Gamma_{s}=\mathbb{1}_{(n+l+m) \times(n+l)}$, solving the optimisation problem in (25) leads to an $\mathscr{H}_{2}$ norm of 69.0439 (Case 1). We then use Algorithm 1 with $\kappa=0.1, \epsilon=0.0001$ and exploit the row-sparsity and column-sparsity promoting functions (37) and (37) and (42), respectively, with the update rules in (38) and (43). By increasing $\eta_{a}$ and $\eta_{s}$ from zero, the number of non-zero rows and non-zero columns of $\tilde{\mathscr{K}}_{a}$ and $\tilde{\mathscr{K}}_{s}$, respectively, decreases. For example, letting $\eta_{a}=500$ and $\eta_{s}=100$, the algorithm suggests exploiting only $\delta_{s p 9-12}$ and $\delta_{r}$ as the control surfaces whilst all 4 sensors' signal are employed in the control system (Case 2). The $\mathscr{H}_{2}$ norm of $T_{w z}$ is 71.6807 , which is about $4 \%$ worse than that obtained by the non-structured feedback gain. Further with $\eta_{a}=100$ and $\eta_{s}=10^{5}$, the result is to use two control surfaces $\left(\delta_{s p 9-12}\right.$ and $\left.\delta_{r}\right)$ as well as 3 sensors (roll rate $p$, sideslip angle $\beta$, and roll angle $\phi$ ) among 4 available sensors (Case 3 ), although with about $25 \%$ increase in the $\mathscr{H}_{2}$-norm of the closed-loop 
(a)

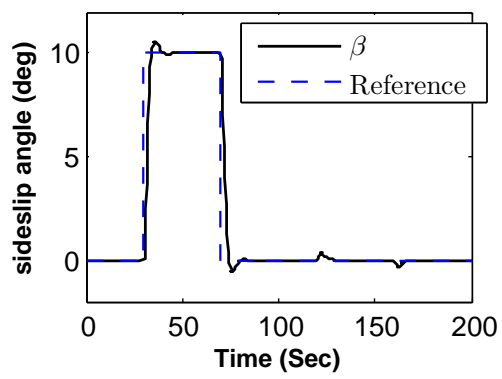

(c)

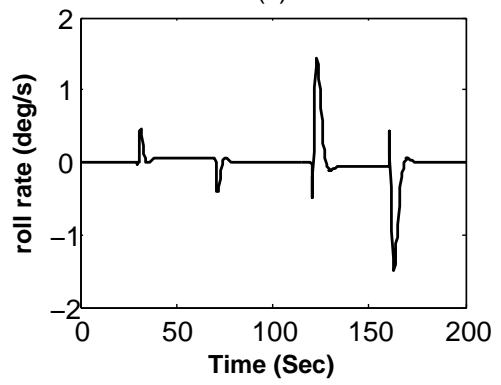

(b)

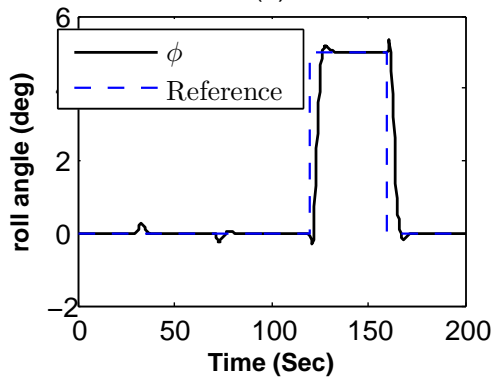

(d)

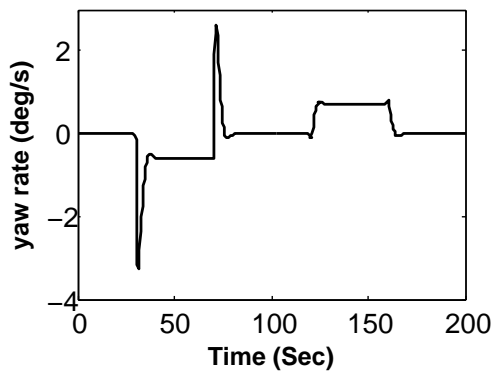

Figure 2: $\quad$ System states for Case 1

system. Note that once a pattern is identified, we need to turn to the problem in (25) to obtain a suboptimal structured DOF controller.

Considering a step change of 10 degree for $\beta$ during 30 to $70 s$ as well as a step change of 5 degree for $\phi$ during 120 to $160 \mathrm{~s}$, Figures 2 24 and 6 8 show the tracking responses of the system for Cases 1-3.

Now, let us consider the case where the rudder is missing. We repeat the above-mentioned procedure, with the difference that a large initial value is assigned to the weight associated with the rudder in the sparsification algorithm (or equivalently a large value is assigned to $\phi_{u, 9}^{-1}$ in the associated performance output $z(t))$. Solving the REL1 algorithm with $\eta_{a}=500$ and $\eta_{s}=10^{5}$, the finding is that $\delta_{s p 9-12}$ and $e_{4}$ can be used for the $\beta$ and $\phi$ tracking problem, in the absence of the rudder, while 3 sensors (roll rate $p$, sideslip angle $\beta$, and roll angle $\phi)$ are exploited (Case 4). However, the performance degradation in this case is about $7 \%$ worse than that of Case 3. Figures 5, 6, 7 and 8 show the tracking responses of the system in this case.

\section{Conclusions}

This paper develops a framework for addressing the issue of selecting an optimal set of actuators (sensors) for dynamical systems, satisfying several performance and pole clustering constraints. Firstly, an LMI-based framework for the design 
(a)

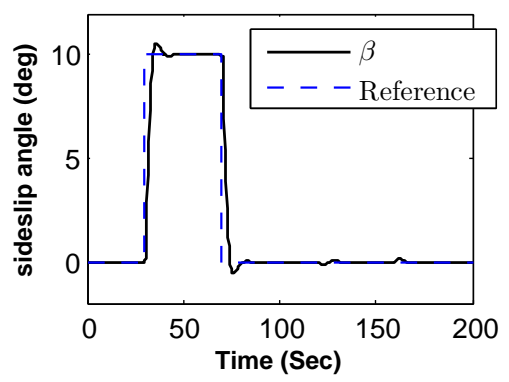

(c)

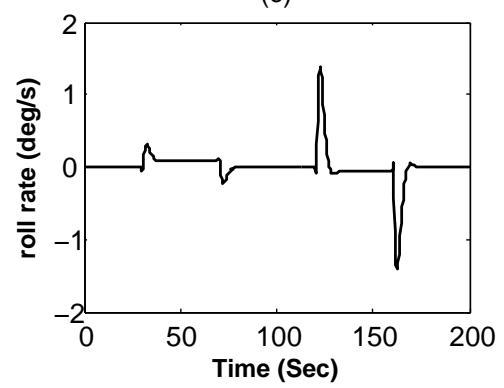

(b)

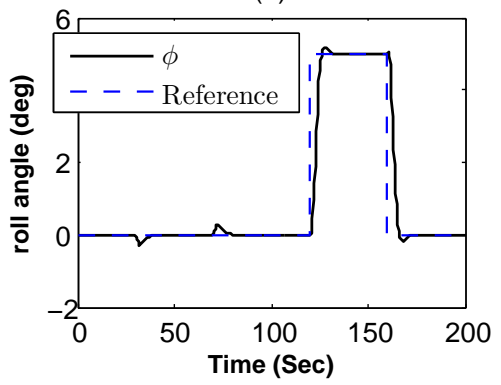

(d)

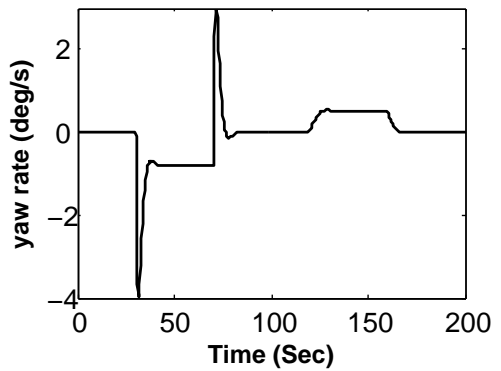

Figure 3: System states for Case 2

(a)

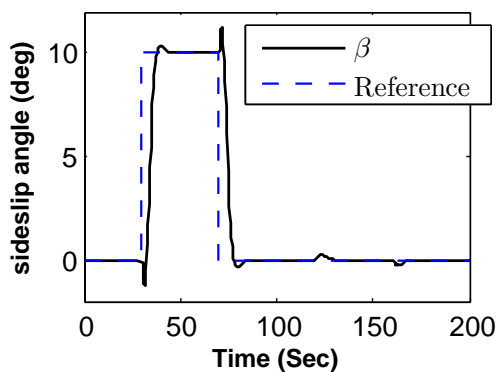

(c)

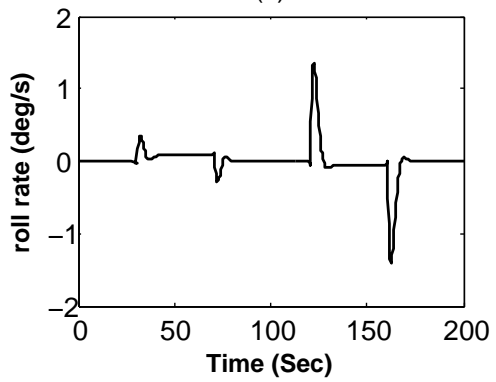

(b)

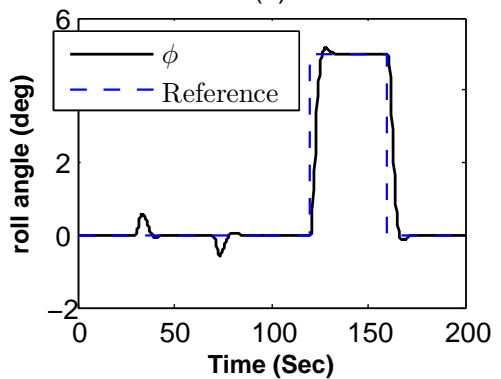

(d)

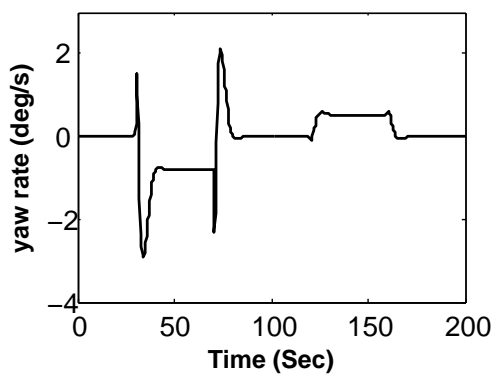

Figure 4: $\quad$ System states for Case 3 
(a)

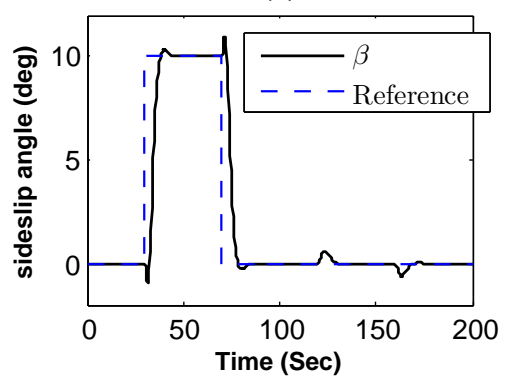

(c)

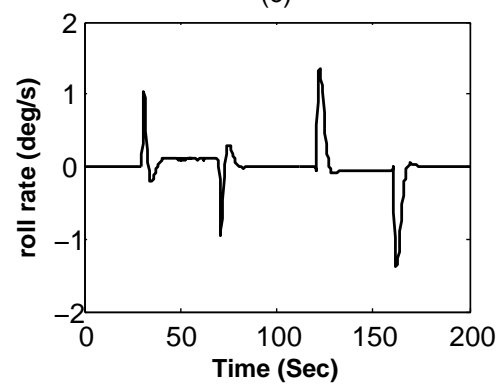

(b)

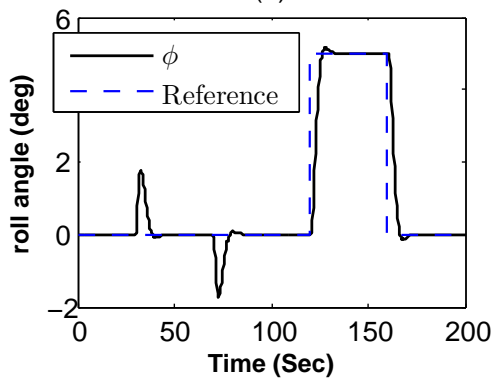

(d)

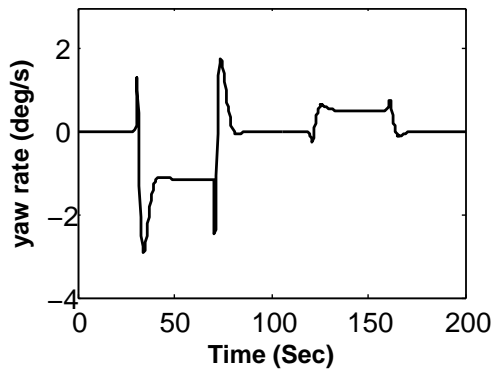

Figure 5: System states for Case 4

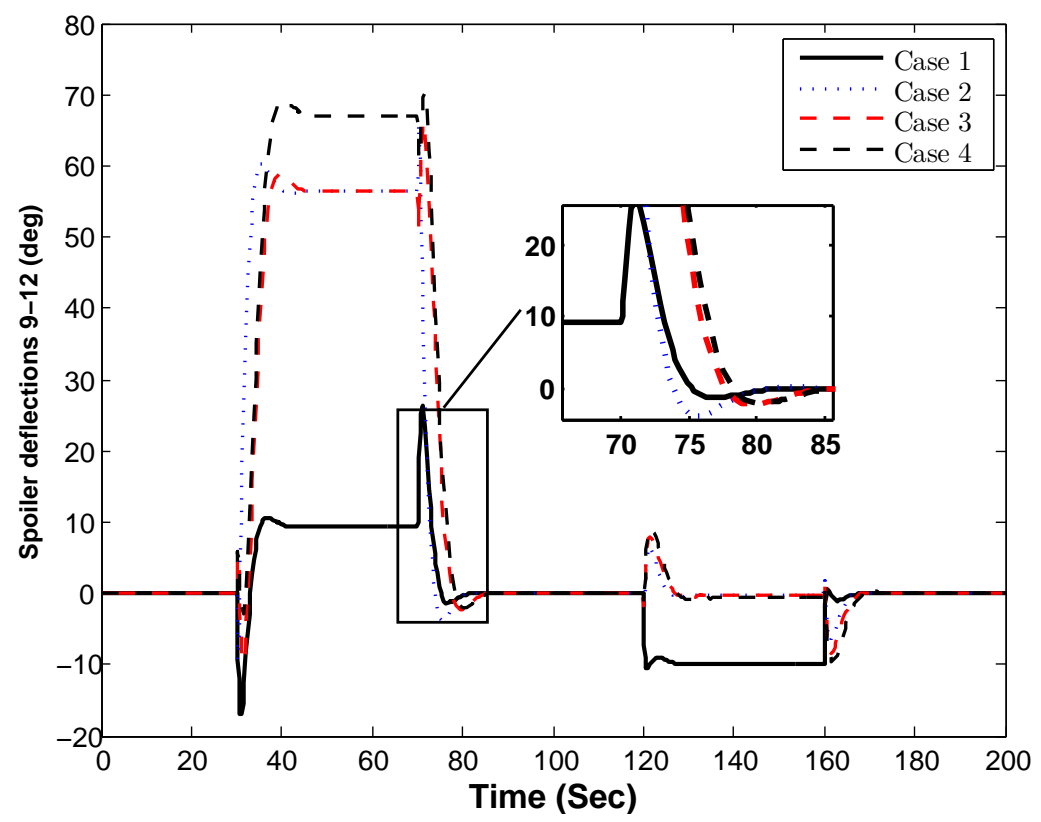

Figure 6: Spoiler deflections 9-12 (deg) 


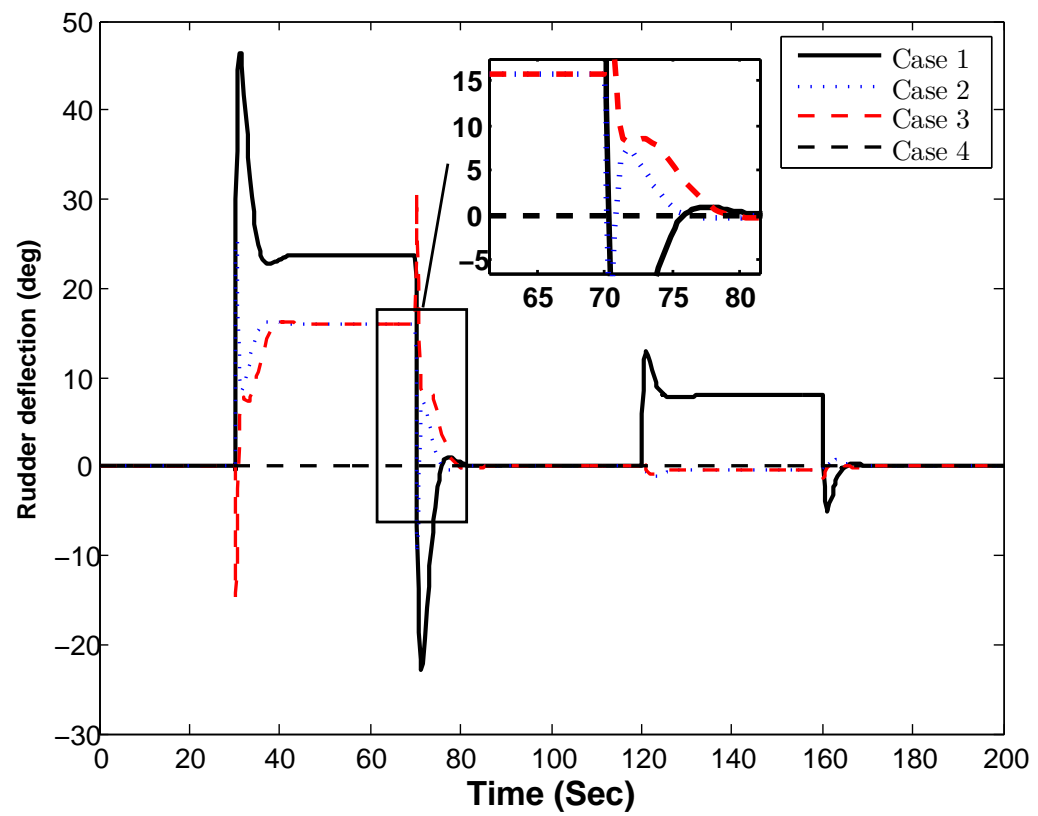

Figure 7: Rudder deflection (deg)

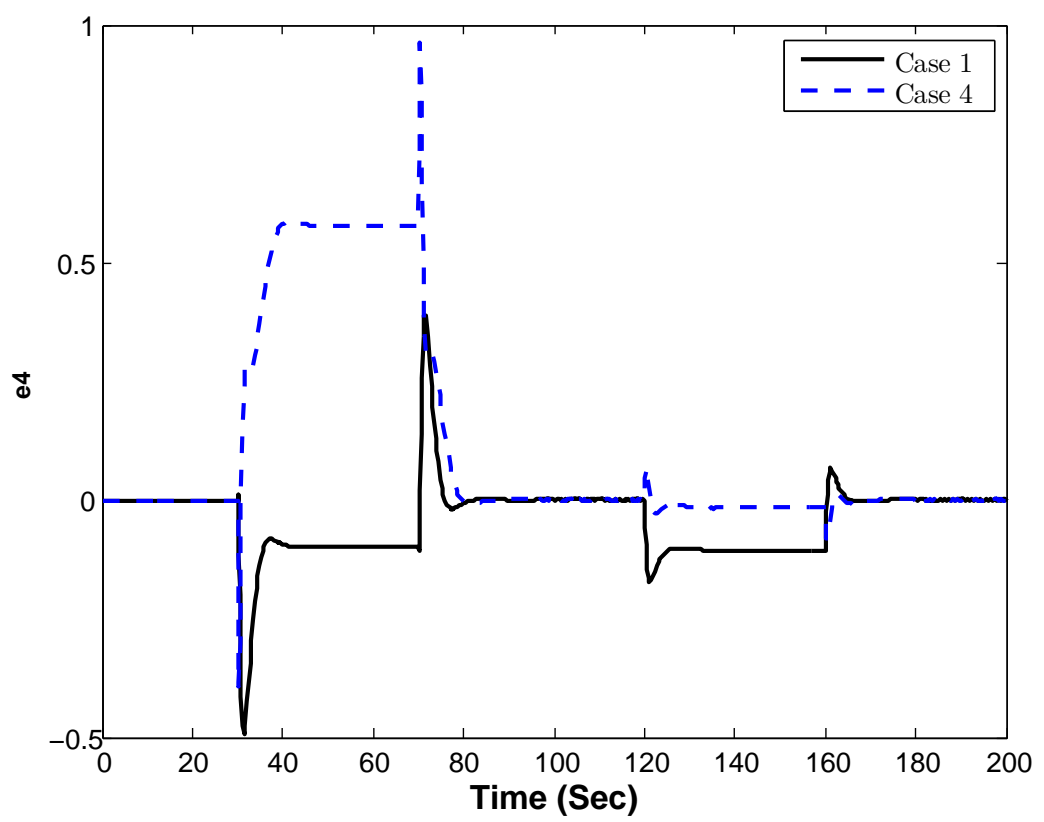

Figure 8: EPR 4 
of multi-channel $\mathscr{H}_{2}$ DOF gain has been proposed. Besides, it has been discussed that this framework is capable of imposing additional convex regional pole placement constraints as well as the structural constraints on the DOF. Following this, a procedure has been developed which includes two stages; the first stage identifies the desirable row and column sparsity pattern for necessary parts of dynamic feedback gain via iterative processes and then the second one solves the multi-channel $\mathscr{H}_{2}$ problem, augmented by structural constraints and/or regional pole placement constraints. This scheme has an immediate application in over-actuated/sensed systems for selecting a subset of available actuators/sensors aimed at minimising power or fuel consumption and/or actuator wear and tear, maintenance cost, etc. The proposed approach has also been shown that has the potential to be employed as a novel fault accommodation scheme searching for a sparse structure for the controller while minimising the performance degradation and allocation error. It is worth noting that it is nevertheless hard to give guarantees on the maximum number of iterations and computation time that the algorithm requires to achieve the optimal solution. This is indeed a common issue in the existing optimisation based fault tolerant control and component selection approaches. Hence, one may resort to accept some degree of sub-optimality for meeting the restrictions of computational resources so that the real-time computation constraints can be satisfied. Additionally, this paper does not involve the Lyapunov function used for checking system performances in the controller variables. This can help us to avoid the unnecessary conservatism of the so-called quadratic approach, and thus can widen the applicability region of the proposed scheme for multi-objective synthesis. However, it may also increase the computation time, since more decision variables are involved in the optimisation-based controller design problem. Nevertheless, in practice it is not necessary to solve the proposed REL1 algorithm exactly and it can be terminated after a few iterations. This feature can significantly reduce the computation time, albeit at the expense of accepting some degree of sub-optimality. Two practical case studies, including the lateral control design for an over-actuated flight, clearly demonstrate the effectiveness of our proposed approaches. However, it should be emphasised that LMI-based approaches are not scalable and solving large LMIs for many iterations may practically limit the size of the underlying problem. A more scalable method such as alternation direction method of multipliers (ADMM) or first-order optimisation methods can be considered in future work. Moreover, in this manuscript, while an optimal scheme has been used to design sparse row/column wise controllers, no limit for rate saturations or actuator magnitude is explicitly imposed on the problem. Theoretically, in the case of exceeding a position limit or rate limit, the occurred difference between the commanded actuator and expected one is thought of as a fault. The proposed optimal scheme can then redistribute the control commands to other actuators, so that the effect of the actuator saturation is mitigated. Nevertheless, in terms of dealing with actuator saturations, imposing explicit constraints on the optimal control synthesis scheme makes the whole problem more practical. This issue can be evaluated in future work. 


\section{References}

Alwi, H., \& Edwards, C. (2010). Fault tolerant control using sliding modes with on-line control allocation. In Fault tolerant flight control (pp. 247-272). Springer.

Apkarian, P., Tuan, H. D., \& Bernussou, J. (2001). Continuous-time analysis, eigenstructure assignment, and $\mathrm{H}_{2}$ synthesis with enhanced linear matrix inequalities (LMI) characterizations. Automatic Control, IEEE Transactions on, 46(12), 1941-1946.

Boyd, S., Ghaoui, L. E., Feron, E., \& Balakrishnan, V. (1994). Linear matrix inequalities in system and control theory. Philadelphia: Society for Industrial and Applied Mathematics.

Candes, E. J., Wakin, M. B., \& Boyd, S. P. (2008). Enhancing sparsity by reweighted $\ell_{1}$ minimization. Journal of Fourier analysis and applications, 14(5-6), 877-905.

Casavola, A., \& Garone, E. (2010). Fault-tolerant adaptive control allocation schemes for overactuated systems. International Journal of Robust and Nonlinear Control, 20(17), 1958-1980.

Chilali, M., \& Gahinet, P. (1996). $H_{\infty}$ design with pole placement constraints: An LMI approach. Automatic Control, IEEE Transactions on, 41(3), 358367.

Chilali, M., Gahinet, P., \& Apkarian, P. (1999). Robust pole placement in LMI regions. Automatic Control, IEEE Transactions on, 44(12), 2257-2270.

Cristofaro, A., \& Johansen, T. A. (2014). Fault tolerant control allocation using unknown input observers. Automatica, 50(7), 1891-1897.

De Oliveira, M., Gerome, J., \& Bernussou, J. (1999). An LMI optimization approach to multiobjective controller design for discrete-time systems. In Decision and control, 1999. proceedings of the 38th ieee conference on (Vol. 4, pp. 3611-3616).

de Oliveira, M. C., Bernussou, J., \& Geromel, J. C. (1999). A new discrete-time robust stability condition. Systems \& control letters, 37(4), 261-265.

Dhingra, N. K., Jovanović, M. R., \& Luo, Z.-Q. (2014). An ADMM algorithm for optimal sensor and actuator selection. In 53rd ieee conference on decision and control (pp. 4039-4044).

Edwards, C., \& Tan, C. P. (2006). Sensor fault tolerant control using sliding mode observers. Control Engineering Practice, 14(8), 897-908.

Fahroo, F., \& Demetriou, M. A. (2000). Optimal actuator/sensor location for active noise regulator and tracking control problems. Journal of Computational and Applied Mathematics, 114(1), 137-158.

Fardad, M., \& Jovanovic, M. R. (2014). On the design of optimal structured and sparse feedback gains via sequential convex programming. In American control conference (acc), 2014 (pp. 2426-2431).

Gahinet, P., \& Apkarian, P. (1993). An lmi-based parametrization of all $\mathscr{H}_{\infty}$ controllers with applications. In Proceedings of the 32nd ieee conference on decision and control (pp. 656-661).

Güney, M., \& Eşkinat, E. (2008). Optimal actuator and sensor placement 
in flexible structures using closed-loop criteria. Journal of Sound and Vibration, 312(1), 210-233.

Härkegård, O., \& Glad, S. T. (2005). Resolving actuator redundancy - optimal control vs. control allocation. Automatica, 41(1), 137-144.

Johansen, T. A., \& Fossen, T. I. (2013). Control allocation-a survey. Automatica, 49(5), 1087-1103.

Joshi, S., \& Boyd, S. (2009). Sensor selection via convex optimization. Signal Processing, IEEE Transactions on, 57(2), 451-462.

Kekatos, V., Giannakis, G. B., \& Wollenberg, B. (2012). Optimal placement of phasor measurement units via convex relaxation. IEEE Transactions on Power Systems, 27(3), 1521-1530.

Leibfritz, F., \& Lipinski, W. (2003). Description of the benchmark examples in COMPleib 1.0 (Tech. Rep.). Trier, Germany: University of Trier.

Leite, V., \& Peres, P. (2003). An improved LMI condition for robust $D$-stability of uncertain polytopic systems. IEEE Transactions on Automatic Control, 48(3), 500-504.

Lin, F., Fardad, M., \& Jovanovic, M. (2011). Augmented Lagrangian approach to design of structured optimal state feedback gains. IEEE Trans. Autom. Control, 56(12), 2923-2929.

Löfberg, J. (2004, September). YALMIP: A toolbox for modeling and optimization in MATLAB. In Cca/isic/cacsd. Retrieved from http://control.ee . ethz.ch/index. cgi?action=details; id=2088; page=publications

Montagner, V. F., \& Peres, P. L. (2005). $\mathscr{H}_{\infty}$ parameter-dependent state feedback control of linear time-varying systems in polytopic domains. In Decision and control, 2005 and 2005 european control conference. cdc-ecc'05. 44th ieee conference on (pp. 5006-5011).

Moreno, C. P., Pfifer, H., \& Balas, G. J. (2015). Actuator and sensor selection for robust control of aeroservoelastic systems. In 2015 american control conference (acc) (pp. 1899-1904).

Nestorović, T., \& Trajkov, M. (2013). Optimal actuator and sensor placement based on balanced reduced models. Mechanical Systems and Signal Processing, 36(2), 271-289.

Peaucelle, D., Arzelier, D., Bachelier, O., \& Bernussou, J. (2000). A new robust $D$-stability condition for real convex polytopic uncertainty. Systems \& Control Letters, 40(1), 21-30.

Polyak, B., Khlebnikov, M., \& Shcherbakov, P. (2013). An LMI approach to structured sparse feedback design in linear control systems. In Control conference (ecc), 2013 european (pp. 833-838).

Rogers, J. (2000). A parallel approach to optimum actuator selection with a genetic algorithm. In Aiaa guidance, navigation, and control conference and exhibit (p. 4484).

Roy, V., Chepuri, S. P., \& Leus, G. (2013). Sparsity-enforcing sensor selection for DOA estimation. In Computational advances in multi-sensor adaptive processing (camsap), 2013 ieee 5th international workshop on (pp. 340$343)$.

Savkin, A. V., \& Evans, R. J. (2002). Hybrid dynamical systems: controller 
and sensor switching problems. Springer Science \& Business Media.

Savkin, A. V., Evans, R. J., \& Skafidas, E. (2001). The problem of optimal robust sensor scheduling. Systems \& Control Letters, 43(2), 149-157.

Scherer, C., Gahinet, P., \& Chilali, M. (1997). Multiobjective output-feedback control via LMI optimization. IEEE Transactions on Automatic Control, $42(7), 896-911$.

Schuler, S., Münz, U., \& Allgöwer, F. (2014). Decentralized state feedback control for interconnected systems with application to power systems. Journal of Process Control, 24(2), 379-388.

Staroswiecki, M., \& Amani, A. M. (2014). Fault-tolerant control of distributed systems by information pattern reconfiguration. International Journal of Adaptive Control and Signal Processing.

Tan, C. P., \& Edwards, C. (2003). Sliding mode observers for robust detection and reconstruction of actuator and sensor faults. International Journal of Robust and Nonlinear Control, 13(5), 443-463.

Toh, K. C., Todd, M., Tütüncü, R., \& Tutuncu, R. H. (1998). SDPT3 - a MATLAB software package for semidefinite programming. Optimization Methods and Software, 11, 545-581.

Van De Wal, M., \& De Jager, B. (2001). A review of methods for input/output selection. Automatica, 37(4), 487-510.

Westermayer, C., Schirrer, A., Hemedi, M., \& Kozek, M. (2009). An advanced criterion for optimal actuator and sensor placement on complex flexible structures. IFAC Proceedings Volumes, 42(2), 114-119.

Zhang, Y., \& Jiang, J. (2002). Active fault-tolerant control system against partial actuator failures. IEE proceedings-Control Theory and applications, 149(1), 95-104.

Zoltowski, D. M., Dhingra, N., Lin, F., \& Jovanovic, M. R. (2014). Sparsitypromoting optimal control of spatially-invariant systems. In American control conference (acc), 2014 (pp. 1255-1260).

\section{A Proof of Lemma 2.1}

The equivalence between the first two statements is a standard $\mathscr{H}_{2}$ state feedback synthesis and can be seen e.g. in Boyd, Ghaoui, Feron, and Balakrishnan (1994). We just show the equivalence between the statements ii) and iii). Exploiting the well-known Schur complement, one can show that the first LMI in iii) can be reformulated as

$$
\left[\begin{array}{cc}
-\left(G+G^{T}\right)+\gamma^{-1}\left(C_{c l} G\right)^{T}\left(C_{c l} G\right) & \star \\
A_{c l} G+X+G & -2 X
\end{array}\right]<0 .
$$


Note that as $G^{T}+G>0, G$ is invertible. By performing congruence transformation $\left[\begin{array}{cc}G^{-T} & 0 \\ 0 & X^{-1}\end{array}\right]$ in the above inequality, we obtain

$$
\left[\begin{array}{cc}
-\left(\tilde{G}+\tilde{G}^{T}\right)+\gamma^{-1} C_{c l}^{T} C_{c l} & \star \\
\tilde{X} A_{c l}+\tilde{X}+\tilde{G} & -2 \tilde{X}
\end{array}\right]<0 .
$$

where $\tilde{G}=G^{-1}$ and $\tilde{X}=X^{-1}$. The above inequality can be written as

$$
\left[\begin{array}{cc}
\gamma^{-1} C_{c l}^{T} C_{c l} & \star \\
\tilde{X} A_{c l}+\tilde{X} & -2 \tilde{X}
\end{array}\right]+\operatorname{herm}\left(\left[\begin{array}{c}
-I \\
I
\end{array}\right] \tilde{G}\left[\begin{array}{ll}
I & 0
\end{array}\right]\right)<0 .
$$

According to the well-known Projection lemma (Gahinet \& Apkarian, 1993), the above inequality holds iff the following inequalities, with respect to $\boldsymbol{G}$, are satisfied:

$$
\begin{aligned}
& {\left[\begin{array}{l}
I \\
I
\end{array}\right]^{T}\left[\begin{array}{cc}
\gamma^{-1} C_{c l}^{T} C_{c l} & \star \\
\tilde{X} A_{c l}+\tilde{X} & -2 \tilde{X}
\end{array}\right]\left[\begin{array}{c}
I \\
I
\end{array}\right]<0,} \\
& {\left[\begin{array}{l}
0 \\
I
\end{array}\right]^{T}\left[\begin{array}{cc}
\gamma^{-1} C_{c l}^{T} C_{c l} & \star \\
\tilde{X} A_{c l}+\tilde{X} & -2 \tilde{X}
\end{array}\right]\left[\begin{array}{l}
0 \\
I
\end{array}\right]<0 .}
\end{aligned}
$$

As seen the inequality $(53)$ indeed becomes the trivial inequality $-\tilde{X}<0$ and (52) is equivalent to

$$
\tilde{X} A_{c l}+A_{c l}^{T} \tilde{X}+\gamma^{-1} C_{c l}^{T} C_{c l}<0 .
$$

With pre- and post-multiplying the above inequality by $X=\tilde{X}^{-1}$ we have

$$
A_{c l} X+X A_{c l}^{T}+\gamma^{-1} X C_{c l}^{T} C_{c l} X<0,
$$

The above inequality can simply be written as item ii), using the Schur complement.

\section{B Proof of Theorem 2.3}

Using the Schur complement, the LMI in 14 can be reformulated as

$$
\left[\begin{array}{cc}
-I_{\xi} \otimes\left(G+G^{T}\right)+\left(I_{\xi} \otimes G^{T}\right)\left(\Xi \otimes X^{-1}\right)\left(I_{\xi} \otimes G\right) & \star \\
\Pi \otimes(\mathscr{A} G)+I_{\xi} \otimes X+I_{\xi} \otimes G & -2 I_{\xi} \otimes X
\end{array}\right]<0 .
$$

Note that as $I_{\xi} \otimes\left(G+G^{T}\right)>0, I_{\xi} \otimes G$ is invertible. By performing congruence transformation $\left[\begin{array}{cc}I_{\xi} \otimes G^{-T} & 0 \\ 0 & I_{\xi} \otimes X^{-1}\end{array}\right]$ in the above inequality, we obtain

$$
\left[\begin{array}{cc}
-I_{\xi} \otimes\left(\tilde{G}+\tilde{G}^{T}\right)+\Xi \otimes \tilde{X} & \star \\
\Pi \otimes(\tilde{X} \mathscr{A})+I_{\xi} \otimes \tilde{X}+I_{\xi} \otimes \tilde{G} & -2 I_{\xi} \otimes \tilde{X}
\end{array}\right]<0 .
$$


where $I_{\xi} \otimes \tilde{G}=I_{\xi} \otimes G^{-1}, \tilde{X}=X^{-1}$. The above inequality can be rewritten as

$$
\left[\begin{array}{cc}
\Xi \otimes \tilde{X} & \star \\
\Pi \otimes(\tilde{X} \mathscr{A})+I_{\xi} \otimes \tilde{X} & -2 I_{\xi} \otimes \tilde{X}
\end{array}\right]+\operatorname{herm}\left(\left[\begin{array}{c}
-I \\
I
\end{array}\right]\left(I_{\xi} \otimes \tilde{G}\right)\left[\begin{array}{ll}
I & 0
\end{array}\right]\right)<0 .
$$

Based on the Projection lemma, the above inequality holds if the following inequalities, with respect to $I_{\xi} \otimes \tilde{G}$, are satisfied:

$$
\begin{gathered}
{\left[\begin{array}{l}
I \\
I
\end{array}\right]^{T}\left[\begin{array}{cc}
\Xi \otimes \tilde{X} & \star \\
\Pi \otimes(\tilde{X} \mathscr{A})+I_{\xi} \otimes \tilde{X} & -2 I_{\xi} \otimes \tilde{X}
\end{array}\right]\left[\begin{array}{l}
I \\
I
\end{array}\right]<0,} \\
{\left[\begin{array}{l}
0 \\
I
\end{array}\right]^{T}\left[\begin{array}{cc}
\Xi \otimes \tilde{X} & \star \\
\Pi \otimes(\tilde{X} \mathscr{A})+I_{\xi} \otimes \tilde{X} & -2 I_{\xi} \otimes \tilde{X}
\end{array}\right]\left[\begin{array}{l}
0 \\
I
\end{array}\right]<0 .}
\end{gathered}
$$

As seen the inequality (55) indeed becomes the trivial inequality $-\tilde{X}<0$ and (54) is equivalent to

$$
\Xi \otimes \tilde{X}+\Pi \otimes(\tilde{X} \mathscr{A})+\Pi \otimes\left(\mathscr{A}^{T} \tilde{X}\right)<0,
$$

Pre- and post-multiplying the above inequality by $I_{\xi} \otimes X=I_{\xi} \otimes\left(\tilde{X}^{-1}\right)$ will lead to

$$
\Xi \otimes X+\Pi \otimes(\mathscr{A} X)+\Pi \otimes\left(X \mathscr{A}^{T}\right)<0 .
$$

This completes the proof.

\section{Proof of Theorem 2.4}

The equivalence between 1) and 3) is shown in e.g. Chilali and Gahinet (1996). Moreover, the equivalence between 2) and 3) is simply obtained through applying the Schur complement with respect to the block $(2,2)$ in 20 . Applying the Schur complement with respect to the block $(1,1)$ in $(21),(19)$ is recovered by choosing $G=G^{T}=\frac{1}{r} X>0$, hence 2) implies 4). Also, left- and rightmultiplication of $(21)$ by $[I \mathscr{A}]$ and $[I \mathscr{A}]^{T}$, respectively, lead to $(19)$. Hence, 4) implies 2), and the proof is completed. 